\title{
POSITIVE CUBATURE FORMULAS AND MARCINKIEWICZ-ZYGMUND INEQUALITIES ON SPHERICAL CAPS
}

\author{
FENG DAI AND HEPING WANG
}

AbStRACT. Let $\Pi_{n}^{d}$ denote the space of all spherical polynomials of degree at most $n$ on the unit sphere $\mathbb{S}^{d}$ of $\mathbb{R}^{d+1}$, and let $d(x, y)$ denote the usual geodesic distance $\arccos x \cdot y$ between $x, y \in \mathbb{S}^{d}$. Given a spherical cap

$$
B(e, \alpha)=\left\{x \in \mathbb{S}^{d}: \quad d(x, e) \leq \alpha\right\}, \quad\left(e \in \mathbb{S}^{d}, \quad \alpha \in(0, \pi) \text { is bounded away from } \pi\right),
$$

we define the metric

$$
\rho(x, y):=\frac{1}{\alpha} \sqrt{(d(x, y))^{2}+\alpha(\sqrt{\alpha-d(x, e)}-\sqrt{\alpha-d(y, e)})^{2}},
$$

where $x, y \in B(e, \alpha)$. It is shown that given any $\beta \geq 1,1 \leq p<\infty$ and any finite subset $\Lambda$ of $B(e, \alpha)$ satisfying the condition $\min _{\substack{\xi, \eta \in \Lambda \\ \xi \neq \eta}} \rho(\xi, \eta) \geq \frac{\delta}{n}$ with $\delta \in(0,1]$, there exists a positive constant $C$, independent of $\alpha, n, \Lambda$ and $\delta$, such that, for any $f \in \Pi_{n}^{d}$,

$$
\sum_{\omega \in \Lambda}\left(\max _{x, y \in B_{\rho}(\omega, \beta \delta / n)}|f(x)-f(y)|^{p}\right)\left|B_{\rho}(\omega, \delta / n)\right| \leq(C \delta)^{p} \int_{B(e, \alpha)}|f(x)|^{p} d \sigma(x),
$$

where $d \sigma(x)$ denotes the usual Lebesgue measure on $\mathbb{S}^{d}$,

$$
B_{\rho}(x, r)=\{y \in B(e, \alpha): \quad \rho(y, x) \leq r\}, \quad(r>0)
$$

and

$$
\left|B_{\rho}\left(x, \frac{\delta}{n}\right)\right|=\int_{B_{\rho}(x, \delta / n)} d \sigma(y) \sim \alpha^{d}\left[\left(\frac{\delta}{n}\right)^{d+1}+\left(\frac{\delta}{n}\right)^{d} \sqrt{1-\frac{d(x, e)}{\alpha}}\right] .
$$

As a consequence, we establish positive cubature formulas and Marcinkiewicz-Zygmund inequalities on the spherical cap $B(e, \alpha)$.

Key words and phrases. Spherical caps, cubature formulas, Marcinkiewicz-Zygmund inequalities, spherical polynomials.

Research started while the second author visited Edmonton. The first author was partially supported by the NSERC Canada under grant G121211001. The second author was partially supported by the NNSF China (No. 10201021), the SRCP Beijing (No. KM200310028106), and the NSF Beijing (No. 1062004). 


\section{INTRODUCTION}

Let $\mathbb{S}^{d}=\left\{x=\left(x_{1}, x_{2}, \cdots, x_{d+1}\right) \in \mathbb{R}^{d+1}: \quad|x|:=\sqrt{x_{1}^{2}+x_{2}^{2}+\cdots+x_{d+1}^{2}}=1\right\}$ denote the unit sphere of $\mathbb{R}^{d+1}$ endowed with the usual rotation invariant measure $d \sigma(x)$. We denote by $d(x, y)$ the geodesic distance $\arccos x \cdot y$ between $x$ and $y$ on $\mathbb{S}^{d}$, by $B(x, r)$ the spherical cap $\left\{y \in \mathbb{S}^{d}: \quad d(x, y) \leq r\right\}$ centered at $x \in \mathbb{S}^{d}$ of radius $r>0$, and by $B(x ; \alpha, \alpha+\underline{)}$ the spherical collar $\left\{y \in \mathbb{S}^{d}: \quad \alpha \leq d(x, y) \leq \alpha+\underline{\}}\right.$ centered at $x \in \mathbb{S}^{d}$ of spherical height $\underline{\underline{i}} 0$. A function on $\mathbb{S}^{d}$ is called a spherical polynomial of degree at most $n$ if it is the restriction to $\mathbb{S}^{d}$ of a polynomial in $d+1$ variables of total degree at most $n$. We denote by $\Pi_{n}^{d}$ the space of all spherical polynomials of degree at most $n$ on $\mathbb{S}^{d}$. Given a set $E$, we shall use the notations \#E, $|E|$ and $\chi_{E}$ to denote its cardinality, Lebesgue measure, and characteristic function, respectively. Moreover, we shall write $A \sim B$ for the statement $C^{-1} \leq A / B \leq C$, where $C>0$ is called the constant of equivalence.

Let $e$ be a fixed point on $\mathbb{S}^{d}, \alpha \in(0, \pi), 1 \leq p<\infty, n$ be a positive integer, and let $\Lambda$ be a finite subset of the spherical cap $B(e, \alpha)$. We are particularly interested in the following question:

Question: What condition on the finite subset $\Lambda$ guarantees the existence of a sequence of positive numbers $\lambda_{\omega}, \omega \in \Lambda$ for which the following two equations hold for all $f \in \Pi_{n}^{d}$ ?

$$
\int_{B(e, \alpha)} f(y) d \sigma(y)=\sum_{\omega \in \Lambda} \lambda_{\omega} f(\omega)
$$

and

$$
\int_{B(e, \alpha)}|f(x)|^{p} d \sigma(x) \sim \sum_{\omega \in \Lambda} \lambda_{\omega}|f(\omega)|^{p},
$$

where the constant of equivalence is independent of $n, f$, and $\alpha$.

In the above question, we would expect a sharp estimate on the weights $\lambda_{\omega}$ and that $\# \Lambda \sim \operatorname{dim} \Pi_{n}^{d}$ as $n \rightarrow \infty$. It should be pointed out that our interest in the above question is motivated by the work [M1] of Mhaskar and the work [KL2, GLN] of Golinskii, Kobindarajah, Lubinsky and Nevai.

Note that the integrals in (1.1) and (1.2) are over the spherical cap $B(e, \alpha)$ rather than the whole sphere $\mathbb{S}^{d}$. An equality like (1.1) with positive weights $\lambda_{\omega}$ is called a positive cubature formula of degree $n$, while an equivalence like (1.2) is called a Marcinkiewicz-Zygmund (MZ) type inequality.

In one dimensional case, MZ inequalities over arcs of the circle for the full range of $0<$ $p<\infty$ were obtained in the paper [GLN, Theorem 1.1] of Golinskii, Lubinsky, and Nevai, and in a more recent paper [KL2] of Kobindarajah and Lubinsky (see Remark 1.5 below for more details). In the case of $d \geq 2$, existence of positive cubature formulas based on scattered data on spherical caps were proved by Mhaskar in [M1] (see Remark 1.6 below for details.) Many useful cubature formulas on $\mathbb{S}^{d}$ with different properties were previously constructed by many authors. For relevant results on $\mathbb{S}^{d}$, one may consult [BD, BDS, D2, GS, MNW, M2, NPW1, NPW2, Pe, Xu1, Xu2], among others.

Here we recall some known results on $\mathbb{S}^{d}$ that are relevant to our current discussion. Positive cubature formulas and MZ inequalities for $1 \leq p \leq \infty$ based on function values at scattered sites on $\mathbb{S}^{d}$ were first established by Mhaskar, Narcowich and Ward in the fundamental paper $\left[\mathrm{MNW}\right.$. Positive cubature formulas on $\mathbb{S}^{d}$ with sharp estimates on the weights were obtained 
by Narcowich, Petrushev and Ward in a more recent paper [NPW1]. A different proof of MZ inequalities on $\mathbb{S}^{d}$ was given in $[\mathrm{BD}]$. Compared with that of $[\mathrm{MNW}]$, the proof in $[\mathrm{BD}]$ works for the full range of $0<p<\infty$ and for all compact two-point homogeneous manifolds. For results concerning doubling weights on $\mathbb{S}^{d}$, we refer to [D1] (in the case $d \geq 2$ ) and the remarkable work [MT2] of Mastroianni and Totik (in the case $d=1$ ).

It should be pointed out that all known proofs of the MZ inequalities on $\mathbb{S}^{d}$ are based on the following integral representation of spherical polynomials:

$$
f(x)=\int_{\mathbb{S}^{d}} f(y) K_{n}(x \cdot y) d \sigma(y), \quad x \in \mathbb{S}^{d}, \quad f \in \Pi_{n}^{d},
$$

where $K_{n}$ is a smooth reproducing kernel for the space $\Pi_{n}^{d}$ ( see, for instance, [BD, (2.13)]). Since the integral in (1.3) is over the whole sphere rather than on a local spherical cap, we find it difficult to use (1.3) to deduce similar results on local spherical caps. In our opinion, in order to obtain an ideal result on a spherical cap $B(e, \alpha)$, special efforts have to be made to treat the center $e$ as well as the boundary of $B(e, \alpha)$. Our proof will be different from those for $\mathbb{S}^{d}$ (see, for instance, $[\mathrm{MNW}, \mathrm{BD}])$. It is based on some recent results obtained in [BD] and [D1], as well as the weighted Markov-Bernstein-type inequality recently proved by Erdèlyi [Er2], rather than the integral representation (1.3).

To state our main results, we need to introduce several necessary notation. Let $\left(X, d_{X}\right)$ be a metric space. We denote by $B_{d_{X}}(x, r)$ the ball $\left\{y \in X: \quad d_{X}(x, y) \leq r\right\}$ centered at $x \in X$ of radius $r>0$. Given $\varepsilon>0$ and a finite subset $A$ of $X$, we say $A$ is $\left(\varepsilon, d_{X}\right)$-separable if it satisfies the condition $\min _{\xi \xi^{\prime} \in A} d_{X}\left(\xi, \xi^{\prime}\right) \geq \varepsilon$; while we say $A$ is maximal $\left(\varepsilon, d_{X}\right)$-separable if it is $\underset{\substack{\xi, \xi^{\prime} \in A \\ \xi \neq \xi^{\prime}}}{ }$

$\left(\varepsilon, d_{X}\right)$-separable and satisfies $X=\bigcup_{\xi \in A} B_{d_{X}}(\xi, \varepsilon)$.

For $x, y \in B(e, \alpha)$, we define

$$
\rho(x, y) \equiv \rho_{B(e, \alpha)}(x, y):=\frac{1}{\alpha} \sqrt{(d(x, y))^{2}+\alpha\left(b_{x}^{1 / 2}-b_{y}^{1 / 2}\right)^{2}},
$$

where $b_{x} \equiv b_{x, B(e, \alpha)}$ denotes the shortest distance from $x \in B(e, \alpha)$ to the boundary of $B(e, \alpha)$; that is

$$
b_{x} \equiv b_{x, B(e, \alpha)}=\alpha-d(x, e) .
$$

It's easily seen that $\rho$ is a metric on $B(e, \alpha)$. For $r>0$ and $x \in B(e, \alpha)$, we define

$$
\triangle_{r}(x) \equiv \triangle_{r, B(e, \alpha)}(x):=\alpha^{d}\left(r^{d+1}+r^{d} \sqrt{1-\frac{d(x, e)}{\alpha}}\right) .
$$

It will be shown in Section 2 (Lemma 2.2 (iii)) that for any $x \in B(e, \alpha)$ and $r \in(0,1)$,

$$
\left|B_{\rho}(x, r)\right| \sim \triangle_{r}(x),
$$

where and throughout $B_{\rho}(x, r):=\{y \in B(e, \alpha): \rho(y, x) \leq r\}$, and the constant of equivalence is independent of $r, x$, and $\alpha$ when $\alpha$ is bounded away from $\pi$.

For the rest of this section, we assume that $B(e, \alpha)$ is given with $\alpha \in(0, \pi)$ bounded away from $\pi$, and we write $\rho, b_{x}$ and $\triangle_{r}(x)$ for $\rho_{B(e, \alpha)}, b_{x, B(e, \alpha)}$ and $\triangle_{r, B(e, \alpha)}(x)$ respectively.

Now our main result in this paper can be stated as follows. 
Theorem 1.1. If $\delta \in(0,1], \beta \geq 1,1 \leq p<\infty$, and $\Lambda$ is a $(\delta / n, \rho)$-separable subset of $B(e, \alpha)$, then for any $f \in \Pi_{n}^{d}$, we have

$$
\sum_{\omega \in \Lambda}\left(\max _{x, y \in B_{\rho}(\omega, \beta \delta / n)}|f(x)-f(y)|^{p}\right)\left|B_{\rho}(\omega, \delta / n)\right| \leq\left(C_{1} \delta\right)^{p} \int_{B(e, \alpha)}|f(x)|^{p} d \sigma(x),
$$

where $C_{1}$ depends only on $d, p$, and $\beta$.

It can be shown that any maximal $(\delta / n, \rho)$-separable subset $\Lambda$ of $B(e, \alpha)$ must satisfy the condition $\# \Lambda \sim \delta^{-d} \operatorname{dim} \Pi_{n}^{d} \sim\left(\frac{n}{\delta}\right)^{d}$, with the constants of equivalence depending only on $d$. In particular, this means that the number of nodes required in the above theorem is comparable to the dimension of $\Pi_{n}^{d}$ as $n \rightarrow \infty$.

As a consequence of Theorem 1.1, we have the following three useful corollaries.

Corollary 1.2. There exists a constant $\delta_{0} \in(0,1)$ depending only on $d$, such that, for any $\delta \in\left(0, \delta_{0}\right)$ and any maximal $(\delta / n, \rho)$-separable subset $\Lambda$ of $B(e, \alpha)$, there exists a sequence of positive numbers $\lambda_{\omega}, \omega \in \Lambda$ satisfying

$$
\lambda_{\omega} \sim \triangle_{\delta / n}(\omega) \sim\left|B_{\rho}\left(\omega, \frac{\delta}{n}\right)\right|, \quad \omega \in \Lambda
$$

with constants of equivalence depending only on $d$, such that the cubature formula (1.1) holds for all $f \in \Pi_{n}^{d}$.

Corollary 1.2 seems new even in the case $d=1$. It can be deduced from Theorem 1.1 following the standard method in [NPW1].

Corollary 1.3. Given $1 \leq p<\infty$ and an arbitrary finite subset $\Lambda$ of $B(e, \alpha)$, there exists a positive constant $C$ depending only on $p$ and $d$, such that, for any $f \in \Pi_{n}^{d}$,

$$
\sum_{\omega \in \Lambda}|f(\omega)|^{p} \triangle_{1 / n}(\omega) \leq C \tau \int_{B(e, \alpha)}|f(x)|^{p} d \sigma(x),
$$

where $\tau$ is defined by

$$
\tau=\max _{x \in \mathbb{S}^{d}} \#\left(\Lambda \cap B_{\rho}\left(x, \frac{1}{n}\right)\right) .
$$

Corollary 1.4. If $\beta \geq 1,1 \leq p<\infty$, and $\Lambda$ is a maximal $\left(\frac{\delta}{n}, \rho\right)$-separable subset of $B(e, \alpha)$ with $\delta \in\left(0, \frac{1}{4 C_{1}}\right.$ ] and $C_{1}$ the same as in Equation [1.7), then for all $f \in \Pi_{n}^{d}$, we have

$$
\begin{aligned}
\int_{B(e, \alpha)}|f(x)|^{p} d \sigma(x) & \sim\left(\sum_{\omega \in \Lambda}\left(\max _{x \in B_{\rho}(\omega, \beta \delta / n)}|f(x)|^{p}\right) \triangle_{\delta / n}(\omega)\right)^{\frac{1}{p}} \\
& \sim\left(\sum_{\omega \in \Lambda}\left(\min _{x \in B_{\rho}(\omega, \beta \delta / n)}|f(x)|^{p}\right) \triangle_{\delta / n}(\omega)\right)^{\frac{1}{p}},
\end{aligned}
$$

where the constants of equivalence are independent of $f, n, \alpha$ and $\{\omega\}_{\omega \in \Lambda}$.

Remark 1.5. In one dimensional case, the following large sieve inequality was proved by Golinskii, Lubinsky, and Nevai [GLN]:

$$
\sum_{k=1}^{m}\left|P\left(\alpha_{j}\right)\right|^{p} \varepsilon\left(\alpha_{j}\right) \leq C \tau \int_{a}^{b}|P(\theta)|^{p} d \theta
$$


with $C$ independent of $m, n, P, p, a, b,\left\{\alpha_{j}\right\}$. Here $P$ is a trigonometric polynomial of degree $\leq n, 1$

$$
\varepsilon(\theta)=\frac{1}{p n+1}\left(\left|\sin \left(\frac{\theta-a}{2}\right) \sin \left(\frac{\theta-b}{2}\right)\right|+\left(\frac{b-a}{p n+1}\right)^{2}\right)^{1 / 2}
$$

while

$$
\begin{gathered}
0 \leq a \leq \alpha_{1}<\alpha_{2}<\cdots<\alpha_{m} \leq b \leq 2 \pi, \\
\tau=\max _{\theta \in[a, b]} \#\left(\left\{j: \quad \alpha_{j} \in[\theta-\varepsilon(\theta), \theta+\varepsilon(\theta)]\right\}\right),
\end{gathered}
$$

$0<p<\infty$ and $m \geq 1$. A version of (1.9), which has the correct form for all choices of $[a, b]$-whether $b-a$ is very small or close to $2 \pi$, was established in a recent paper [KL2] by Kobindarajah and Lubinsky. Note that if we identify the interval $[a, b]$ with the arc $B(e, \alpha)$ centered at $e=\left(\cos \frac{a+b}{2}, \sin \frac{a+b}{2}\right)$ and of radius $\alpha=\frac{b-a}{2}$, we would have

$$
\varepsilon(\theta) \sim \triangle_{1 / n}(z) \sim\left|B_{\rho}\left(z, \frac{1}{n}\right)\right|,
$$

provided that $b-a$ is not too close to $2 \pi$, where $\theta \in[a, b]$ and $z=(\cos \theta, \sin \theta)$. This means that Corollary 1.3 can be considered as a higher-dimensional analogue of the large sieve inequality (1.9). While we believe Corollary 1.3 remains true for $0<p<1$ as well, we are unable to prove it.

Remark 1.6. It was shown by Mhaskar [M1] that given an arbitrary set $\Lambda$ of points in $B(e, \alpha)$ satisfying the mesh norm condition $\max _{\xi \in \mathbb{S}^{d}} \min _{\omega \in \Lambda} d(\omega, \xi) \leq c \alpha$, there exists nonnegative weights $\lambda_{\omega}, \omega \in \Lambda$ such that (1.1) holds for every $f \in \Pi_{n}^{d}$. Here the constant $c$ is independent of $f$ and $\alpha$, but depends on the degree $n$. Here we wish to compare our result with that of Mhaskar [M1]. First, our results are uniform in the degree $n$ and the radius $\alpha$ (when $\alpha$ is not very close to $\pi$ ), while his result is not uniform in $n$. Indeed, in our opinion, uniformity in the degree is of crucial importance, as can be seen in many known work (see, for instance, [GLN, D2, NPW2]). Second, our result ( Corollary 1.2) shows that the minimum number of nodes required in a positive cubature formula of degree $n$ on $B(e, \alpha)$ is comparable to the dimension of the space $\Pi_{n}^{d}$ as $n \rightarrow \infty$, while his result does not. Third, we have a sharp estimate $\lambda_{\omega} \sim \triangle_{\delta / n}(\omega)$ on the weights of the cubature formula (1.1), while only nonnegativity of the cubature weights were shown in [M1]. Fourth, as mentioned in Remark 1.5, our result can be considered as a higher dimensional analogue of the large sieve inequality of Golinskii, Lubinsky, and Nevai. Fifth, in our work we made special efforts to treat the boundary and the center of the spherical cap, while Mhaskar [M1] didn't. In our opinion, good cubature formulas and MZ inequalities on a spherical cap $B(e, \alpha)$ couldn't be obtained without taking into consideration of the boundary of $B(e, \alpha)$. Indeed, our opinion is supported by many known results on a finite interval $[a, b]$. Last but not least, as is demonstrated in Section 5 (Theorem 5.1), our method can be used to obtain MZ inequalities with doubling weights on spherical caps.

The paper is organized as follows. In Section 2, we show two technical lemmas concerning the properties of the metric $\rho$ in the case when $\alpha \in\left(0, \frac{1}{2}\right]$. After that, we prove the main results, Theorem 1.1 and Corollaries 1.2-1.4, for the case $\alpha \in\left(0, \frac{1}{2}\right]$ in Section 3. The proofs of the main results for the remaining case $\alpha \in\left(\frac{1}{2}, \pi\right)$ can be deduced from the case $\alpha \in\left(0, \frac{1}{2}\right]$. This is done

\footnotetext{
${ }^{1}$ In $[\mathrm{GLN}], P$ could be a " generalized trigonometric polynomial", not just an ordinary trigonometric polynomial.
} 
in Section 4. Finally, in Section 5, we discuss briefly how to establish similar results for spherical collars and for spherical caps with doubling weights.

\section{TWO BASIC LEMMAS}

In this section, we establish some basic facts concerning the metric $\rho \equiv \rho_{B(e, \alpha)}$ defined by (1.4) in the case when $\alpha \in\left(0, \frac{1}{2}\right]$. These facts will be needed in later sections. We shall begin with the simple case $d=1$, where $\mathbb{S}^{1}$ is the unit circle, identified as $\mathbb{R} / 2 \pi \mathbb{Z}$.

Let $\alpha \in\left(0, \frac{1}{2}\right)$. For $x_{1}, x_{2} \in[-\alpha, \alpha]$, we define

$$
\rho_{1}\left(x_{1}, x_{2}\right) \equiv \rho_{[-\alpha, \alpha]}\left(x_{1}, x_{2}\right):=\frac{1}{\alpha} \sqrt{\left|x_{1}-x_{2}\right|^{2}+\alpha\left|b_{x_{1}}^{1 / 2}-b_{x_{2}}^{1 / 2}\right|^{2}}
$$

where $b_{x} \equiv b_{x,[-\alpha, \alpha]}$ denotes the shortest distance from $x \in[-\alpha, \alpha]$ to the boundary of $[-\alpha, \alpha]$, that is,

$$
b_{x} \equiv b_{x,[-\alpha, \alpha]}:=\min \{|x+\alpha|,|x-\alpha|\} .
$$

Clearly, $\rho_{1}$ is the one-dimensional analog of the metric $\rho \equiv \rho_{B(e, \alpha)}$ defined by (1.4). It turns out ( see Lemma 2.1 below) that $\rho_{1}$ is equivalent to two other metrics $\rho_{2}$ and $\rho_{3}$ on $[-\alpha, \alpha]$, whose definitions are given as follows: for $x_{1}=\arcsin \left((\sin \alpha) \cos t_{1}\right)$ and $x_{2}=\arcsin \left((\sin \alpha) \cos t_{2}\right)$ with $t_{1}, t_{2} \in[0, \pi]$,

$$
\begin{aligned}
& \rho_{2}\left(x_{1}, x_{2}\right)=\frac{1}{\alpha} \sqrt{\left|x_{1}-x_{2}\right|^{2}+\left|\sqrt{\alpha^{2}-x_{1}^{2}}-\sqrt{\alpha^{2}-x_{2}^{2}}\right|^{2}}, \\
& \rho_{3}\left(x_{1}, x_{2}\right)=\left|t_{1}-t_{2}\right| .
\end{aligned}
$$

For $x \in[-\alpha, \alpha]$ and $r \in(0,1)$, we write $B_{\rho_{i}}(x, r):=\left\{y \in[-\alpha, \alpha]: \rho_{i}(x, y) \leq r\right\}, i=1,2,3$.

Now our first lemma can be stated as follows:

Lemma 2.1. Let $\alpha \in\left(0, \frac{1}{2}\right.$ ] and let $\rho_{1}, \rho_{2}$ and $\rho_{3}$ be defined by (2.1), (2.2) and (2.3), respectively. Then the following statements hold true:

(i) For any $x_{1}, x_{2} \in[-\alpha, \alpha]$,

$$
\rho_{1}\left(x_{1}, x_{2}\right) \sim \rho_{2}\left(x_{1}, x_{2}\right) \sim \rho_{3}\left(x_{1}, x_{2}\right),
$$

where the constants of equivalence are independent of $\alpha, x_{1}$ and $x_{2}$.

(ii) For any $x \in[-\alpha, \alpha]$ and $r \in(0,1)$, we have

$$
B_{\rho_{1}}(x, r) \subset[x-\alpha r, x+\alpha r] \cap[-\alpha, \alpha]
$$

and

$$
\left|B_{\rho_{i}}(x, r)\right| \sim \alpha\left(r^{2}+r \sqrt{1-(x / \alpha)^{2}}\right), \quad i=1,2,3,
$$

where the constant of equivalence is independent of $x, r$ and $\alpha$.

(iii) For any $x_{1}, x_{2} \in[-\alpha, \alpha]$ and $r>0$,

$$
\left|B_{\rho_{1}}\left(x_{1}, r\right)\right| \leq C\left(1+\frac{\rho_{1}\left(x_{1}, x_{2}\right)}{r}\right)\left|B_{\rho_{1}}\left(x_{2}, r\right)\right|,
$$

where $C>0$ is independent of $x_{1}, x_{2}, r$ and $\alpha$.

(iv) If $r \in(0,1), \beta \geq 1$ and $A$ is an $\left(r, \rho_{1}\right)$-separable subset of $[-\alpha, \alpha]$, then

$$
\sup _{x \in[-\alpha, \alpha]} \sum_{\xi \in A} \chi_{B_{\rho_{1}}(\xi, \beta r)}(x) \leq C \beta^{3},
$$

where $C$ is an absolute constant. 
Proof. (i) First, we show the equivalence

$$
\rho_{1}\left(x_{1}, x_{2}\right) \sim \rho_{2}\left(x_{1}, x_{2}\right) .
$$

To show this, we start with the case $x_{1} x_{2} \geq 0$. Without loss of generality, we may assume in this case that $x_{1}, x_{2} \in[0, \alpha]$ (otherwise, consider $-x_{1}$ and $-x_{2}$ ). Then $b_{x_{i}}=\alpha-x_{i}, i=1,2$, and thus, by the definition, we obtain

$$
\begin{aligned}
& \rho_{1}\left(x_{1}, x_{2}\right) \sim \frac{\left|x_{1}-x_{2}\right|}{\alpha}+\frac{\left|x_{1}-x_{2}\right|}{\sqrt{\alpha}\left(\sqrt{\alpha-x_{1}}+\sqrt{\alpha-x_{2}}\right)}, \\
& \rho_{2}\left(x_{1}, x_{2}\right) \sim \frac{\left|x_{1}-x_{2}\right|}{\alpha}+\frac{\left|x_{1}-x_{2}\right|}{\sqrt{\alpha}\left(\sqrt{\alpha-x_{1}}+\sqrt{\alpha-x_{2}}\right)} \frac{x_{1}+x_{2}}{\alpha} .
\end{aligned}
$$

If $x_{1}+x_{2} \geq \frac{\alpha}{4}$ then $x_{1}+x_{2} \sim \alpha$, so comparison (2.9) with (2.10) gives $\rho_{1}\left(x_{1}, x_{2}\right) \sim \rho_{2}\left(x_{1}, x_{2}\right)$. However, on the other hand, if $x_{1}+x_{2} \leq \frac{\alpha}{4}$ then $\sqrt{\alpha-x_{1}}+\sqrt{\alpha-x_{2}} \sim \sqrt{\alpha}$, so by (2.9) and (2.10), we deduce $\rho_{1}\left(x_{1}, x_{2}\right) \sim \rho_{2}\left(x_{1}, x_{2}\right) \sim \frac{\left|x_{1}-x_{2}\right|}{\alpha}$. This proves (2.8) in the case $x_{1} x_{2} \geq 0$.

Equation (2.8) for the case $x_{1} x_{2}<0$ follows from the case $x_{1} x_{2} \geq 0$. In fact, by the already proven case $x_{1} x_{2} \geq 0$, we deduce that if $x_{1} x_{2}<0$ then $\rho_{1}\left(x_{1},-x_{2}\right) \sim \rho_{2}\left(x_{1},-x_{2}\right)$, which together with (2.1) and (2.2) implies

$$
\frac{\left|x_{1}+x_{2}\right|}{\alpha}+\frac{\left|\sqrt{\alpha^{2}-x_{1}^{2}}-\sqrt{\alpha^{2}-x_{2}^{2}}\right|}{\alpha} \sim \frac{\left|x_{1}+x_{2}\right|}{\alpha}+\frac{\left|b_{x_{1}}^{\frac{1}{2}}-b_{x_{2}}^{\frac{1}{2}}\right|}{\sqrt{\alpha}} .
$$

Note, on the other hand, that if $x_{1} \cdot x_{2} \leq 0$ then $\left|x_{1}+x_{2}\right| \leq\left|x_{1}-x_{2}\right|$. Thus, using (2.11), we conclude that for $x_{1}, x_{2} \in[-\alpha, \alpha]$ with $x_{1} x_{2}<0$,

$$
\frac{\left|x_{1}-x_{2}\right|}{\alpha}+\frac{\left|\sqrt{\alpha^{2}-x_{1}^{2}}-\sqrt{\alpha^{2}-x_{2}^{2}}\right|}{\alpha} \sim \frac{\left|x_{1}-x_{2}\right|}{\alpha}+\frac{\left|b_{x_{1}}^{\frac{1}{2}}-b_{x_{2}}^{\frac{1}{2}}\right|}{\sqrt{\alpha}},
$$

which implies $\rho_{1}\left(x_{1}, x_{2}\right) \sim \rho_{2}\left(x_{1}, x_{2}\right)$, and therefore completes the proof of (2.8).

Next, we show

$$
\rho_{2}\left(x_{1}, x_{2}\right) \sim \rho_{3}\left(x_{1}, x_{2}\right) .
$$

To this end, we set, for $t \in[0, \pi]$,

$$
g(t)=\arcsin ((\sin \alpha) \cos t) \text { and } h(t)=\sqrt{\alpha^{2}-(g(t))^{2}} .
$$

Since $\alpha \in\left(0, \frac{1}{2}\right]$, it is easy to verify that for $t \in[0, \pi]$

$$
g(t) \sim h^{\prime}(t) \sim \alpha \cos t \text { and } h(t) \sim-g^{\prime}(t) \sim \alpha \sin t .
$$

Now we assume that $x_{1}=g\left(t_{1}\right)$ and $x_{2}=g\left(t_{2}\right)$ with $t_{1}, t_{2} \in[0, \pi]$. Then by (2.2),

$$
\begin{aligned}
\rho_{2}\left(x_{1}, x_{2}\right) & \sim \frac{1}{\alpha}\left[\left|g\left(t_{1}\right)-g\left(t_{2}\right)\right|+\left|h\left(t_{1}\right)-h\left(t_{2}\right)\right|\right] \\
& =\frac{1}{\alpha}\left|\int_{I} g^{\prime}(t) d t\right|+\frac{1}{\alpha}\left|\int_{I} h^{\prime}(t) d t\right|,
\end{aligned}
$$

where $I=\left[t_{1}, t_{2}\right]$ or $\left[t_{2}, t_{1}\right]$. By (2.13), we obtain

$$
\rho_{2}\left(x_{1}, x_{2}\right) \leq C|I|=C\left|t_{1}-t_{2}\right|=C \rho_{3}\left(x_{1}, x_{2}\right) .
$$

To show the converse inequality

$$
\rho_{2}\left(x_{1}, x_{2}\right) \geq C\left|t_{1}-t_{2}\right|
$$


we note that if $\left|t_{1}-t_{2}\right| \leq \frac{\pi}{6}$ then by the mean value theorem for integrals, we obtain, for some $\xi_{1}, \xi_{2} \in I$,

$$
\begin{aligned}
\rho_{2}\left(x_{1}, x_{2}\right) & =\frac{|I|}{\alpha}\left(\left|g^{\prime}\left(\xi_{1}\right)\right|+\left|h^{\prime}\left(\xi_{2}\right)\right|\right) \\
& \geq C|I|\left(\left|\sin \xi_{1}\right|+\left|\cos \xi_{2}\right|\right) \quad(\text { by }(\underline{2.13})) \\
& \geq C|I|\left(\sin ^{2} \xi_{1}+\cos ^{2} \xi_{1}-\left|\cos \xi_{2}-\cos \xi_{1}\right|\right) \\
& \geq C|I|\left(1-\left|\xi_{1}-\xi_{2}\right|\right) \geq C\left(1-\frac{\pi}{6}\right)|I| .
\end{aligned}
$$

On the other hand, if $\left|t_{1}-t_{2}\right| \geq \frac{\pi}{6}$, then by (2.13) it follows that

$$
\rho_{2}\left(x_{1}, x_{2}\right) \geq \frac{1}{\alpha}\left|\int_{I} g^{\prime}(t) d t\right| \geq C \int_{I} \sin t d t \geq C|I| .
$$

This completes the proof of (2.12).

(ii) Since by the definition, for all $x_{1}, x_{2} \in[-\alpha, \alpha]$,

$$
\left|x_{1}-x_{2}\right| \leq \alpha \rho_{1}\left(x_{1}, x_{2}\right)
$$

it follows that $B_{\rho_{1}}(x, r) \subset[x-\alpha r, x+\alpha r] \bigcap[-\alpha, \alpha]$. Thus, by (2.4), it remains to show

$$
\left|B_{\rho_{3}}(x, r)\right| \sim \alpha\left(r^{2}+r \sqrt{1-(x / \alpha)^{2}}\right) .
$$

Again, we set $g(t)=\arcsin ((\sin \alpha) \cos t)$. Given $x \in[-\alpha, \alpha]$, we shall use the natation $t_{x}$ to denote the unique solution in $[0, \pi]$ to the equation $g(t)=x$. Then we have

$$
B_{\rho_{3}}(x, r)=\left\{g(t): t \in[0, \pi] \text { and }\left|t-t_{x}\right| \leq r\right\} .
$$

For the proof of (2.15), we start with the case $x \in[0, \alpha]$. In this case, $t_{x} \in\left[0, \frac{\pi}{2}\right]$, and therefore setting $\gamma=\max \left\{0, t_{x}-r\right\}$, we obtain

$$
\begin{aligned}
\left|B_{\rho_{3}}(x, r)\right| & =g(\gamma)-g\left(t_{x}+r\right) \sim \alpha \int_{\gamma}^{t_{x}+r} \sin u d u \\
& \sim \alpha \int_{t_{x}+\frac{r}{2}}^{t_{x}+r} \sin u d u \sim \alpha r\left(t_{x}+r\right),
\end{aligned}
$$

where in the first " " we have used (2.13), while in the second " $\sim$ " we have used the doubling property of the weight function $|\sin t|$ ( see, for instance, [MT2]). On the other hand, by (2.2) and (2.4), we have

$$
t_{x}=\rho_{3}(x, \alpha) \sim \rho_{2}(x, \alpha) \sim \sqrt{1-\frac{x}{\alpha}} \sim \sqrt{1-\left(\frac{x}{\alpha}\right)^{2}},
$$

which combined with (2.17) yields the desired equation (2.15) in the case $x \in[0, \alpha]$.

We conclude the proof of (2.15) by showing that the case $x \in[-\alpha, 0]$ follows from the already proven case $x \in[0, \alpha]$. In fact, since $g(\pi-t)=-g(t)$, we have, for $x \in[-\alpha, 0]$,

$$
\begin{aligned}
B_{\rho_{3}}(x, r) & =\left\{g(t): t \in[0, \pi] \text { and }\left|t-t_{x}\right| \leq r\right\} \\
& =\left\{-g(u): u \in[0, \pi] \text { and }\left|u-t_{-x}\right| \leq r\right\}=-B_{\rho_{3}}(-x, r) .
\end{aligned}
$$

Thus, by the already proven case $x \in[0, \alpha]$, we deduce that for $x \in[-\alpha, 0]$

$$
\left|B_{\rho_{3}}(x, r)\right|=\left|B_{\rho_{3}}(-x, r)\right| \sim \alpha\left(r^{2}+r \sqrt{1-(x / \alpha)^{2}}\right),
$$


which gives (2.15) in this case.

(iii) Note that for $x_{1}, x_{2} \in[-\alpha, \alpha]$,

$$
\left|\frac{\alpha\left(r^{2}+r \sqrt{1-\left(x_{1} / \alpha\right)^{2}}\right)}{\alpha\left(r^{2}+r \sqrt{1-\left(x_{2} / \alpha\right)^{2}}\right)}-1\right|=\frac{\left|\sqrt{\alpha^{2}-x_{1}^{2}}-\sqrt{\alpha^{2}-x_{2}^{2}}\right|}{\alpha r+\sqrt{\alpha^{2}-x_{2}^{2}}} \leq \frac{\rho_{2}\left(x_{1}, x_{2}\right)}{r} .
$$

The desired inequality (2.6) then follows by (2.5) and (2.4).

(iv) Let $\beta \geq 1$ and let $A$ be an $\left(r, \rho_{1}\right)$-separable subset of $[-\alpha, \alpha]$. Then by the definition of $\left(r, \rho_{1}\right)$-separable, it follows that for any $x \in[-\alpha, \alpha]$,

$$
\sum_{\xi \in A \cap B_{\rho_{1}}(x, \beta r)}\left|B_{\rho_{1}}\left(\xi, \frac{r}{4}\right)\right| \leq\left|B_{\rho_{1}}\left(x,\left(\beta+\frac{1}{4}\right) r\right)\right| .
$$

However, on the other hand, by (2.6), we note that for any $\xi \in B_{\rho_{1}}(x, \beta r)$,

$$
(C \beta)^{-1}\left|B_{\rho_{1}}\left(x, \frac{r}{4}\right)\right| \leq\left|B_{\rho_{1}}\left(\xi, \frac{r}{4}\right)\right| \leq C \beta\left|B_{\rho_{1}}\left(x, \frac{r}{4}\right)\right| .
$$

Thus, combining (2.18) with (2.19), we obtain

$$
\sum_{\xi \in A} \chi_{B_{\rho_{1}}(\xi, \beta r)}(x)=\#\left(A \bigcap B_{\rho_{1}}(x, \beta r)\right) \leq C \beta \frac{\left|B_{\rho_{1}}\left(x,\left(\beta+\frac{1}{4}\right) r\right)\right|}{\left|B_{\rho_{1}}\left(x, \frac{r}{4}\right)\right|} \leq C \beta^{3},
$$

which proves (2.7).

The proof of Lemma 2.1 is complete.

Now we turn to the case $d \geq 2$. Recall that $\rho=\rho_{B(e, \alpha)}$ is the metric on the spherical cap $B(e, \alpha)$ defined by (1.4), and $\rho_{1}=\rho_{[-\alpha, \alpha]}$ is the metric on $[-\alpha, \alpha]$ defined by (2.1). We need to introduce two more metrics $\rho_{4}$ and $\rho_{5}$ on $B(e, \alpha)$. To this end, we set, for $e \in \mathbb{S}^{d}$,

$$
\mathbb{S}_{e}^{d-1}:=\left\{y \in \mathbb{S}^{d}: y \cdot e=0\right\} .
$$

For $x=e \cos \theta+\xi \sin \theta$ and $y=e \cos t+\eta \sin t$ with $\xi, \eta \in \mathbb{S}_{e}^{d-1}$ and $\theta, t \in[0, \alpha]$, we define

$$
\begin{gathered}
\rho_{4}(x, y):=\max \left\{\rho_{1}(\theta, t), d(\xi, \eta)\right\}, \\
\rho_{5}(x, y):=\frac{1}{\sin \alpha} \sqrt{|\xi \sin \theta-\eta \sin t|^{2}+\left|\sqrt{\sin ^{2} \alpha-\sin ^{2} \theta}-\sqrt{\sin ^{2} \alpha-\sin ^{2} t}\right|^{2}} .
\end{gathered}
$$

Recall that for $x \in B(x, r), r>0$ and a metric $\widetilde{\rho}$ on $B(e, \alpha)$,

$$
B_{\widetilde{\rho}}(x, r)=\{y \in B(e, \alpha): \quad \widetilde{\rho}(x, y) \leq r\} .
$$

Lemma 2.2. Let $\varepsilon \in(0,1), e \in \mathbb{S}^{d}$ and $\alpha \in\left(0, \frac{1}{2}\right]$. Then the following statements hold true:

$$
\begin{aligned}
& \rho(x, y) \sim \rho_{5}(x, y), \quad \text { for all } x, y \in B(e, \alpha) ; \\
& \rho(x, y) \sim \rho_{4}(x, y), \quad \text { if } x, y \in B(e ; \varepsilon \alpha, \alpha) ; \\
& \rho(x, y) \sim \frac{1}{\alpha} d(x, y), \quad \text { if } x, y \in B(e,(1-\varepsilon) \alpha),
\end{aligned}
$$

where the constants of equivalence are independent of $x, y$ and $\alpha$, but may depend on $\varepsilon$ when $\varepsilon$ is small.

(ii) If $x \in B(e, \alpha)$, then for any $r>0$,

$$
B_{\rho}(x, r) \subset B(x, \alpha r), \quad B_{\rho_{4}}(x, r) \subset B(x, 3 \alpha r) ;
$$


if $x \in B(e ; \varepsilon \alpha, \alpha)$, then for any $r>0$,

$$
B_{\rho_{4}}\left(x, C_{2}^{-1} r\right) \subset B_{\rho}(x, r) \subset B_{\rho_{4}}\left(x, C_{2} r\right) ;
$$

if $x \in B(e,(1-\varepsilon) \alpha)$ then for any $r>0$,

$$
B\left(x, C_{2}^{-1} \alpha r\right) \subset B_{\rho}(x, r) \subset B(x, \alpha r),
$$

where $C_{2}$ is independent of $r, x$ and $\alpha$, but depends on $\varepsilon$ when $\varepsilon$ is small.

(iii) For any $x \in B(e, \alpha)$ and $r \in(0,1)$,

$$
\left|B_{\rho}(x, r)\right| \sim \alpha^{d}\left(r^{d+1}+r^{d} \sqrt{\frac{b_{x}}{\alpha}}\right),
$$

where $b_{x} \equiv b_{x, B(e, \alpha)}$ is defined by (1.5), and the constant of equivalence depends only on $d$.

(iv) For any $x, y \in B(e, \alpha)$ and $r>0$,

$$
\left|B_{\rho}(x, r)\right| \leq C\left(1+\frac{\rho(x, y)}{r}\right)\left|B_{\rho}(y, r)\right|,
$$

where $C>0$ depends only on $d$.

(v) Suppose that $r \in(0,1), \beta \geq 1$ and $\Lambda$ is an $(r, \rho)$-separable subset of $B(e, \alpha)$. Then we have

$$
\max _{x \in B(e, \alpha)} \sum_{\omega \in \Lambda} \chi_{B \rho(\omega, \beta r)}(x) \leq C \beta^{d+2},
$$

where $C>0$ depends only on $d$.

Proof. (i) Let $x=\xi \sin \theta+e \cos \theta$ and $y=\eta \sin t+e \cos t$ with $\xi, \eta \in \mathbb{S}_{e}^{d-1}$ and $\theta, t \in[0, \alpha]$. We start with the proof of (2.22). We first note that

$$
4 \sin ^{2}\left(\frac{d(x, y)}{2}\right)=|x-y|^{2}=4 \sin ^{2} \frac{\theta-t}{2}+(\sin \theta \sin t)|\xi-\eta|^{2},
$$

which implies

$$
d(x, y) \sim|\theta-t|+|\eta-\xi| \sqrt{\theta \cdot t} .
$$

Since $\alpha \in\left(0, \frac{1}{2}\right]$, it follows by a straightforward calculation that

$$
|\xi \sin \theta-\eta \sin t| \sim|\theta-t|+|\eta-\xi| \sqrt{\theta \cdot t} \sim d(x, y),
$$

and

$$
\left|\sqrt{\sin ^{2} \alpha-\sin ^{2} \theta}-\sqrt{\sin ^{2} \alpha-\sin ^{2} t}\right| \sim \frac{(\theta+t)\left|\sqrt{b_{x}}-\sqrt{b_{y}}\right|}{\sqrt{\alpha}},
$$

where $b_{x}=\alpha-\theta$ and $b_{y}=\alpha-t$. On the other hand, note, however, that $\theta+t \in[0,2 \alpha]$ and that

$$
\sqrt{\alpha}\left|\sqrt{b_{x}}-\sqrt{b_{y}}\right|=\frac{\sqrt{\alpha}|\theta-t|}{\sqrt{\alpha-\theta}+\sqrt{\alpha-t}} \sim|\theta-t| \leq C d(x, y),
$$

provided $\theta+t<\frac{\alpha}{2}$. This means that

$$
d(x, y)+\frac{(\theta+t)\left|\sqrt{b_{x}}-\sqrt{b_{y}}\right|}{\sqrt{\alpha}} \sim d(x, y)+\sqrt{\alpha}\left|\sqrt{b_{x}}-\sqrt{b_{y}}\right| .
$$

Therefore, combining (2.33) $-(2.35)$, we deduce the desired equivalence (2.22). 
Next, we show (2.23) in the case when $\theta, t \in[\varepsilon \alpha, \alpha]$. In fact, we have

$$
\begin{aligned}
\rho_{4}(x, y) & \sim|\xi-\eta|+\left[\frac{1}{\alpha}|\theta-t|+\frac{1}{\sqrt{\alpha}}|\sqrt{\alpha-t}-\sqrt{\alpha-\theta}|\right] \\
& \sim \frac{1}{\alpha} d(x, y)+\frac{1}{\sqrt{\alpha}}|\sqrt{\alpha-t}-\sqrt{\alpha-\theta}| \sim \rho(x, y),
\end{aligned}
$$

where in the first " $\sim$ " we have used (2.1) and (2.20), in the second " $~$ " we have used (2.32) and the fact that $\theta, t \in[\varepsilon \alpha, \alpha]$, and the final " $\sim$ " follows by (1.4). This proves the desired equation (2.23).

Finally, we note that (2.24) for $\theta, t \in[0,(1-\varepsilon) \alpha]$ is a simple consequence of the definition (1.4) and the following equation:

$$
\frac{1}{\sqrt{\alpha}}\left|\sqrt{b_{x}}-\sqrt{b_{y}}\right|=\frac{1}{\sqrt{\alpha}} \frac{|\theta-t|}{\sqrt{\alpha-t}+\sqrt{\alpha-\theta}} \sim \frac{1}{\alpha}|\theta-t| \leq \alpha^{-1} d(x, y) .
$$

(ii) It follows by (1.4), (2.20) and (2.31) that

$$
d(x, y) \leq \min \left\{\alpha \rho(x, y), 3 \alpha \rho_{4}(x, y)\right\}, \quad x, y \in B(e, \alpha),
$$

which implies (2.25). Thus, it remains to show (2.26) and (2.27). By the definition, it's easily seen that for all $u, v \in[0, \alpha]$ and $y, z \in B(e, \alpha)$,

$$
\rho_{1}(u, v) \leq 3, \quad \max \left\{\rho(y, z), \rho_{4}(y, z)\right\} \leq \pi .
$$

Thus, without loss of generality, we may assume that $r \in\left(0, \frac{\varepsilon}{6}\right]$. Then taking into account (2.36), we deduce that for $x \in B(e ; \varepsilon \alpha, \alpha)$,

$$
B_{\rho}(x, r) \bigcup B_{\rho_{4}}(x, r) \subset B\left(e ; \frac{\varepsilon \alpha}{2}, \alpha\right),
$$

which together with (2.23) implies (2.26). Finally, Equation (2.27) follows by (2.24) and (2.25).

(iii) We start with the case $\alpha / 6 \leq \theta:=d(x, e) \leq \alpha$. In this case, by (2.26), it is sufficient to show that for $r \in\left(0, \frac{1}{12}\right)$,

$$
\left|B_{\rho_{4}}(x, r)\right| \sim \alpha^{d}\left(r^{d+1}+r^{d} \sqrt{b_{x} / \alpha}\right) .
$$

Notice that by Lemma 2.1 (ii), $B_{\rho_{1}}(\theta, r) \subset\left[\frac{1}{12} \alpha, \alpha\right]$. Thus

$$
\left|B_{\rho_{4}}(x, r)\right| \sim r^{d-1} \int_{B_{\rho_{1}}(\theta, r)} \sin ^{d-1} t d t \sim(\alpha r)^{d-1}\left|B_{\rho_{1}}(\theta, r)\right| .
$$

This last equation together with (2.5) implies (2.37) and hence (2.28) in the case when $\theta=$ $d(x, e) \geq \frac{1}{6} \alpha$.

Finally, we note that (2.28) for the case $0 \leq \theta=d(x, e) \leq \frac{\alpha}{6}$ follows directly from (2.27).

(iv) Inequality (2.29) is a simple consequence of (2.28) and the following equation:

$$
\left|\frac{\sqrt{\alpha} r+\sqrt{b_{x}}}{\sqrt{\alpha} r+\sqrt{b_{y}}}-1\right|=\frac{\left|\sqrt{b_{x}}-\sqrt{b_{y}}\right|}{\sqrt{\alpha} r+\sqrt{b_{y}}} \leq \frac{\sqrt{\alpha} \rho(x, y)}{\sqrt{\alpha} r+\sqrt{b_{y}}} \leq \frac{\rho(x, y)}{r} .
$$

(v) (2.30) follows by (2.28), (2.29) and the standard volume comparison method. Since the proof is almost identical to that of Lemma 2.1 (iv), we omit the details.

This completes the proof of Lemma 2.2 . 


\section{Proofs of the main Results For $\alpha \in\left(0, \frac{1}{2}\right]$}

The proofs of Theorem 1.1 and Corollaries $1.2-1.4$ in the case when $\alpha \in\left(0, \frac{1}{2}\right]$ are based on a series of lemmas. To state these lemmas, we need to introduce several notations. We say a weight function $W$ on $\mathbb{S}^{d}$ is a doubling weight if there exists a constant $L$, called doubling constant, such that for all $x \in \mathbb{S}^{d}$ and $r \in(0, \pi)$,

$$
W(B(x, 2 r)) \leq L W(B(x, r)),
$$

where and elsewhere, we write, for a subset $E$ of $\mathbb{S}^{d}$,

$$
W(E)=\int_{E} W(y) d \sigma(y) .
$$

As usual, we identify the unit circle $\mathbb{S}^{1}$ with $\mathbb{R} / 2 \pi \mathbb{Z}$. Thus, $\Pi_{n}^{1} \equiv \Pi_{n}\left(\mathbb{S}^{1}\right)$ denotes the space of all trigonometric polynomials of degree at most $n$ on $\mathbb{R}$. Associated with a function $f$ on $[-\alpha, \alpha]$, we define

$$
f_{\alpha}(t)=f(\arcsin ((\sin \alpha) \cos t)), \quad t \in[-\pi, \pi],
$$

and associated with a weight function $W$ on $[-\alpha, \alpha]$, we define

$$
W_{n, \alpha}(t):=n \int_{t-\frac{1}{n}}^{t+\frac{1}{n}} W_{\alpha}(\theta) d \theta, \quad n=1,2, \cdots
$$

Our first lemma is due to T. Erdélyi [Er2, Theorems 1.3 and 2.1]:

Lemma 3.1. ([Er2]). Let $p \in[1, \infty)$ and $\alpha \in\left(0, \frac{1}{2}\right]$. Suppose $W$ is a weight function on $[-\alpha, \alpha]$ such that $W_{\alpha}$ is a doubling weight on $\mathbb{S}^{1}$. Then for all $T \in \Pi_{n}^{1}$, we have

$$
\int_{-\alpha}^{\alpha}\left|T^{\prime}(t)\right|^{p} W(t)\left(\frac{\alpha}{n}+\sqrt{\alpha^{2}-t^{2}}\right)^{p} d t \leq C n^{p} \int_{-\alpha}^{\alpha}|T(t)|^{p} W(t) d t,
$$

and

$$
\int_{-\pi}^{\pi}\left|T_{\alpha}(t)\right|^{p} W_{\alpha}(t)|\sin t| d t \sim \int_{-\pi}^{\pi}\left|T_{\alpha}(t)\right|^{p} W_{n, \alpha}(t)|\sin t| d t
$$

where the constant $C$ and the constant of equivalence depend only on $p$ and the doubling constant of $W_{\alpha}$.

It was pointed out in [Er2] that $W_{\alpha}$ is a doubling weight if and only if $W(\alpha \cos t)$ is a doubling weight. In the unweighted case, (3.1) for all $0<p<\infty$ was proved by Lubinsky [L] ( see also the paper [KL1] by Kobindarajah and Lubinsky ). For relevant results concerning doubling weights, one may consult [D1, Er1,Er2, MT1, MT2, MT3].

To state our next lemma, we recall that $\rho_{1}=\rho_{[-\alpha, \alpha]}$ is the metric on $[-\alpha, \alpha]$ defined by (2.1).

Lemma 3.2. Let $\alpha \in\left(0, \frac{1}{2}\right], \beta \geq 1,1 \leq p<\infty$ and $\delta \in(0,1)$. Let $W$ be a weight function on $[-\alpha, \alpha]$ such that $W_{\alpha}$ is a doubling weight on $\mathbb{S}^{1}$. Suppose that $n$ is a positive integer and $\left\{\xi_{j}\right\}_{j=1}^{m_{n}}$ is a $\left(\frac{\delta}{n}, \rho_{1}\right)$-separable subset of $[-\alpha, \alpha]$. Then for any $T \in \Pi_{n}^{1}$, we have

$$
\sum_{i=1}^{m_{n}}\left(\max _{x, y \in B_{\rho_{1}}\left(\xi_{i}, \frac{\beta \delta}{n}\right)}|T(x)-T(y)|^{p}\right) \int_{B_{\rho_{1}}\left(\xi_{i}, \frac{\delta}{n}\right)} W(t) d t \leq\left(C \beta^{2} \delta\right)^{p} \int_{-\alpha}^{\alpha}|T(x)|^{p} W(x) d x,
$$

where $C>0$ depends only on $p$ and the doubling constant of $W_{\alpha}$. 
Proof. As in the proof of Lemma 2.1, we set

$$
g(t) \equiv g(t, \alpha)=\arcsin ((\sin \alpha) \cos t), \quad t \in[-\pi, \pi] .
$$

The proof is based on Lemma 3.1. Let $n_{1}=[n / \delta], T \in \Pi_{n}^{1}$, and $1 \leq p<\infty$. Suppose that

$$
\left\{\xi_{j}=g\left(t_{j}\right): t_{j} \in[0, \pi], \quad j=1,2, \cdots m_{n}\right\}
$$

is $\left(\frac{\delta}{n}, \rho_{1}\right)$-separable in $[-\alpha, \alpha]$. Then by Lemma 2.1 (i), there exists an absolute constant $\gamma \geq 1$ such that

$$
\min _{1 \leq i \neq j \leq m_{n}}\left|t_{i}-t_{j}\right| \geq \frac{\delta}{\gamma n}
$$

and for all $1 \leq j \leq m_{n}$ and $r>0$,

$$
B_{\rho_{1}}\left(\xi_{j}, r\right) \subset\left\{g(t): t \in\left[t_{j}-\gamma r, t_{j}+\gamma r\right] \cap[0, \pi]\right\} .
$$

It follows that for a fixed $j \in\left[1, m_{n}\right]$ and any $\beta \geq 1$,

$$
\begin{aligned}
& \left(\max _{x, y \in B_{\rho_{1}}\left(\xi_{j}, \frac{\beta \delta}{n}\right)}|T(x)-T(y)|^{p}\right)\left(\int_{B_{\rho_{1}}\left(\xi_{j}, \frac{\delta}{n}\right)} W(\xi) d \xi\right) \\
& \leq C \alpha^{p+1}\left(\int_{t_{j}-\frac{\gamma \beta \delta}{n}}^{t_{j}+\frac{\gamma \beta \delta}{n}}\left|T^{\prime}(g(t))\right|^{p}|\sin t| d t\right)\left(\int_{t_{j}-\frac{\gamma \delta}{n}}^{t_{j}+\frac{\gamma \delta}{n}} W_{\alpha}(t)|\sin t| d t\right)\left(\int_{t_{j}-\frac{\gamma \beta \delta}{n}}^{t_{j}+\frac{\gamma \beta \delta}{n}}|\sin t| d t\right)^{p-1} \\
& \leq C\left(\frac{\gamma \beta \delta}{n}\right)^{p-1} \alpha^{p+1} \min _{t \in\left[t_{j}-\frac{\gamma \beta \delta}{n}, t_{j}+\frac{\gamma \beta \delta}{n}\right]}\left(|\sin t|+\frac{4 \beta \gamma \delta}{n}\right)^{p} \\
& \quad \times\left(\int_{t_{j}-\frac{\gamma \beta \delta}{n}}^{t_{j}+\frac{\gamma \beta \delta}{n}}\left|T^{\prime}(g(t))\right|^{p}|\sin t| d t\right)\left(\int_{t_{j}-\frac{\gamma \delta}{n}}^{t_{j}+\frac{\gamma \delta}{n}} W_{\alpha}(t) d t\right) \\
& \leq C \beta^{2 p-1} \alpha^{p+1}\left(\frac{\delta}{n}\right)^{p} \int_{t_{j}-\frac{\gamma \beta \delta}{n}}^{t_{j}+\frac{\gamma \beta \delta}{n}}\left|T^{\prime}(g(t))\right|^{p}|\sin t|\left(|\sin t|+\frac{1}{n}\right)^{p} W_{n_{1}, \alpha}(t) d t,
\end{aligned}
$$

where in the first inequality we have used (2.13), (3.4) and Hölder's inequality, and in the last inequality we have used the doubling property of $W_{\alpha}$. Thus, by (3.3), we deduce

$$
\begin{aligned}
& \sum_{j=1}^{m_{n}}\left(\max _{x, y \in B_{\rho_{1}}\left(\xi_{j}, \frac{\beta \delta}{n}\right)}|T(x)-T(y)|^{p}\right)\left(\int_{B_{\rho_{1}}\left(\xi_{j}, \frac{\delta}{n}\right)} W(\xi) d \xi\right) \\
& \quad \leq C \beta^{2 p} \alpha^{p+1}\left(\frac{\delta}{n}\right)^{p} \int_{0}^{\pi}\left|T^{\prime}(g(t))\right|^{p} \sin t\left(\sin t+\frac{1}{n}\right)^{p} W_{n_{1}, \alpha}(t) d t \\
& \left.\quad \sim \beta^{2 p}\left(\frac{\delta}{n}\right)^{p} \int_{-\alpha}^{\alpha}\left|T^{\prime}(x)\right|^{p}\left(\sqrt{\alpha^{2}-x^{2}}+\frac{\alpha}{n}\right)^{p} \widetilde{W}(x) d x \equiv: I, \quad \text { (by (2.13) }\right)
\end{aligned}
$$

where $\widetilde{W}(x)=W_{n_{1}, \alpha}(\arccos (\sin x / \sin \alpha))$. Note that $\widetilde{W}_{\alpha}(t) \equiv \widetilde{W}(g(t))=W_{n_{1}, \alpha}(t)$ and that $W_{n_{1}, \alpha}(t)$ is a doubling weight on $\mathbb{S}^{1}$ with the doubling constant depending only on that of $W_{\alpha}$. It follows by (2.13) and Lemma 3.1 that

$$
\begin{aligned}
I & \leq\left(C \beta^{2} \delta\right)^{p} \int_{-\alpha}^{\alpha}|T(x)|^{p} \widetilde{W}(x) d x \sim \beta^{2 p} \delta^{p} \alpha \int_{0}^{\pi}\left|T_{\alpha}(t)\right|^{p} W_{n_{1}, \alpha}(t) \sin t d t \\
& \sim \beta^{2 p} \delta^{p} \alpha \int_{0}^{\pi}\left|T_{\alpha}(t)\right|^{p} W_{\alpha}(t) \sin t d t \sim \beta^{2 p} \delta^{p} \int_{-\alpha}^{\alpha}|T(x)|^{p} W(x) d x .
\end{aligned}
$$


This completes the proof of Lemma 3.2.

Lemma 3.3. Let $W$ be a doubling weight on $\mathbb{S}^{d}$. Let $\delta \in(0,1), \beta \geq 1$ and $0<p<\infty$. Suppose that $n$ is a positive integer and $\Lambda \subset \mathbb{S}^{d}$ is $\frac{\delta}{n}$-separable with respect to the geodesic metric $d(\cdot, \cdot)$ on $\mathbb{S}^{d}$. Then for all $f \in \Pi_{n}^{d}$,

$$
\sum_{\omega \in \Lambda}\left(\max _{x \in B\left(\omega, \frac{\beta \delta}{n}\right)}|f(x)|^{p}\right) W(B(\omega, \delta / n)) \leq C \int_{\mathbb{S}^{d}}|f(x)|^{p} W(x) d \sigma(x),
$$

and

$$
\sum_{\omega \in \Lambda}\left(\max _{x, y \in B\left(\omega, \frac{\beta \delta}{n}\right)}|f(x)-f(y)|^{p}\right) W(B(\omega, \delta / n)) \leq(C \delta)^{p} \int_{\mathbb{S}^{d}}|f(x)|^{p} W(x) d \sigma(x),
$$

where $C$ depends only on $d, \beta, p$ and the doubling constant of $W$.

Proof. Equation (3.5) is a direct consequence of Equation (3.6). Equation (3.6) with $\beta=1$ was proved in [D1, Corollary 3.3], and the proof there works equally well for $\beta>1$.

Our fourth lemma is due to Mhaskar, Narcowich and Ward [MNW, Proposition 4.1]. Let $X$ be a finite dimensional normed linear space, $X^{*}$ be its dual, and $Z \subset X^{*}$ be a finite subset with cardinality $m$. We say $Z$ is a norming set for $X$ if the operator $x \mapsto\left(y^{*}(x)\right)_{y^{*} \in Z}$ from $X$ to $\mathbb{R}^{m}$ is injective. A functional $x^{*} \in X^{*}$ is said to be positive with respect to $Z$ if for all $x \in X$, $x^{*}(x) \geq 0$ whenever $\min _{y^{*} \in Z} y^{*}(x) \geq 0$.

Lemma 3.4. ([MNW]). Let $X$ be a finite dimensional normed linear space, $X^{*}$ be its dual, $Z \subset X^{*}$ be a finite, norming set for $X$, and $x^{*} \in X^{*}$ be positive with respect to $Z$. Suppose further that $\sup _{x \in X} \min _{y^{*} \in Z} y^{*}(x)>0$. Then there exists a sequence of nonnegative numbers $\ell_{y^{*}}$, $\left(y^{*} \in Z\right)$ such that for any $x \in X$,

$$
x^{*}(x)=\sum_{y^{*} \in Z} \ell_{y^{*}} y^{*}(x) .
$$

Recall that for $0<a<b \leq \pi$ and $e \in \mathbb{S}^{d}$,

$$
B(e ; a, b)=\left\{y \in \mathbb{S}^{d}: \quad a \leq d(e, y) \leq b\right\} .
$$

Our final lemma, Lemma 3.5 below, will play a crucial role in the proof of Theorem 1.1.

Lemma 3.5. Let $\beta \geq 1, \alpha \in\left(0, \frac{1}{2}\right]$ and $1 \leq p<\infty$. Let $\Lambda$ be a $\left(\frac{\delta}{n}, \rho\right)$-separable subset of $B(e, \alpha)$. Then for all $f \in \Pi_{n}^{d}$,

$$
\sum_{\omega \in \Lambda \cap B\left(e ; \frac{\alpha}{12}, \alpha\right)}\left(\max _{x, y \in B_{\rho}\left(\omega, \frac{\beta \delta}{n}\right)}|f(x)-f(y)|^{p}\right)\left|B_{\rho}\left(\omega, \frac{\delta}{n}\right)\right| \leq(C \delta)^{p} \int_{B(e, \alpha)}|f(x)|^{p} d \sigma(x),
$$

where $C>0$ depends only on $d, p$ and $\beta$.

For the moment, we take Lemma 3.5 for granted and proceed with the proof of our main results.

Proof of Theorem 1.1. Let $1 \leq p<\infty, \beta \geq 1, f \in \Pi_{n}^{d}$ and let $\Lambda$ be a $\left(\frac{\delta}{n}, \rho\right)$-separable subset of $B(e, \alpha)$. Set $\Lambda_{1}=\Lambda \bigcap B\left(e, \frac{\alpha}{12}\right)$. Then by Lemma 3.5, it will suffice to show that

$$
\sum_{\omega \in \Lambda_{1}}\left(\max _{x, y \in B_{\rho}\left(\omega, \frac{\beta \delta}{n}\right)}|f(x)-f(y)|^{p}\right)\left|B_{\rho}\left(\omega, \frac{\delta}{n}\right)\right| \leq(C \delta)^{p} \int_{B(e, \alpha)}|f(x)|^{p} d \sigma(x) .
$$


For the proof of (3.7), we take $u \in B(e, \alpha)$ so that $d(u, e)=\frac{\alpha}{6}$. Then associated with the spherical cap $B(u, \alpha / 2)$, we define

$$
\widetilde{\rho}(x, y) \equiv \rho_{B(u, \alpha / 2)}(x, y)=\frac{2}{\alpha} \sqrt{(d(x, y))^{2}+\frac{\alpha}{2}\left|\sqrt{\widetilde{b}_{x}}-\sqrt{\widetilde{b}_{y}}\right|^{2}},
$$

where $x, y \in B\left(u, \frac{\alpha}{2}\right)$, and $\widetilde{b}_{x} \equiv b_{x, B(u, \alpha / 2)}$ denotes the shortest distance from $x \in B(u, \alpha / 2)$ to the boundary of $B\left(u, \frac{\alpha}{2}\right)$. Since

$$
\Lambda_{1} \subset B\left(e, \frac{\alpha}{12}\right) \subset B\left(u ; \frac{\alpha}{12}, \frac{\alpha}{4}\right),
$$

by Lemma 2.2 applied to both $\rho \equiv \rho_{B(e, \alpha)}$ and $\widetilde{\rho} \equiv \rho_{B(u, \alpha / 2)}$, we conclude that the following statements hold true:

$$
\begin{aligned}
\widetilde{\rho}(x, y) & \sim \rho(x, y) \sim \frac{d(x, y)}{\alpha}, \quad \text { for any } x, y \in \Lambda_{1}, \quad(\text { by (2.24) }) \\
\left|B_{\rho}(\omega, r)\right| & \sim\left|B_{\widetilde{\rho}}(\omega, r)\right| \sim(\alpha r)^{d}, \quad \text { for any } \omega \in \Lambda_{1} \text { and } r \in(0,1), \quad(\text { by (2.28) }) \\
B_{\rho}(\omega, r) & \left.\subset B_{\widetilde{\rho}}\left(\omega, 2 C_{2} r\right), \quad \text { for any } \omega \in \Lambda_{1} \text { and } r>0, \quad \text { (by (2.27) }\right)
\end{aligned}
$$

where all constants of equivalence depend only on $d$, and $C_{2}$ is the absolute constant in Equation (2.27) with $\varepsilon=\frac{1}{2}$. By Equation (3.8), we know that there exists an absolute constant $\gamma \in(0,1)$ such that $\Lambda_{1}$ is $\left(\frac{\gamma \delta}{n}, \widetilde{\rho}\right)$-separable in $B(u, \alpha / 2)$. However, on the other hand, using (3.9) and (3.10), we deduce that the sum on the left-hand side of (3.7) is controlled by

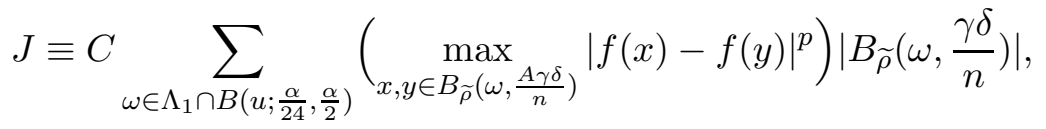

where $A=2 \beta C_{2} / \gamma$. Therefore, by Lemma 3.5 applied to $B\left(u, \frac{\alpha}{2}\right)$ and $\widetilde{\rho} \equiv \rho_{B(u, \alpha / 2)}$, it follows that

$$
J \leq C(\gamma \delta)^{p} \int_{B(u, \alpha / 2)}|f(x)|^{p} d \sigma(x) \leq(C \delta)^{p} \int_{B(e, \alpha)}|f(x)|^{p} d \sigma(x),
$$

which proves the desired equation (3.7) and hence (1.7).

Now we turn to the proofs of Corollaries.

Proof of Corollary 1.2. Let $C_{1}$ denote the constant in (1.7) with $\beta=p=1$. Let $\delta \in\left(0, \frac{1}{4 C_{1}}\right]$, $n_{1}=\left[n /\left(4 C_{1} \delta\right)\right]$ and let $\Lambda$ be a maximal $\left(\frac{\delta}{n}, \rho\right)$-separable subset of $B(e, \alpha)$. We shall prove that there exists a sequence of positive numbers $\lambda_{\omega}, \omega \in \Lambda$ such that $\lambda_{\omega} \sim\left|B_{\rho}\left(\omega, \frac{\delta}{n}\right)\right|$ and (1.1) holds for all $f \in \Pi_{n_{1}}^{d}$. The idea of our proof below is from [NPW].

Note, by Lemma $2.2(\mathrm{v})$, that

$$
1 \leq B(x) \equiv \sum_{\omega \in \Lambda} \chi_{B \rho\left(\omega, \frac{\delta}{n}\right)}(x) \leq C, \quad x \in B(e, \alpha),
$$

where $C \geq 1$ depends only on $d$. We define the following linear functional on $\Pi_{n_{1}}^{d}$ :

$$
\ell(f)=2 \int_{B(e, \alpha)} f(x) d \sigma(x)-\sum_{\omega \in \Lambda}\left(\int_{B_{\rho}\left(\omega, \frac{\delta}{n}\right)} \frac{d \sigma(x)}{B(x)}\right) f(\omega), \quad f \in \Pi_{n_{1}}^{d} .
$$


We then claim that there exists a sequence of nonnegative numbers $\mu_{\omega}, \omega \in \Lambda$ such that

$$
\ell(f)=\sum_{\omega \in \Lambda} \mu_{\omega} f(\omega), \quad \text { for all } f \in \Pi_{n_{1}}^{d} .
$$

For the proof of the claim (3.12), we note that, by (1.7), each $f \in \Pi_{n_{1}}^{d}$ is uniquely determined by its restriction to the set $\Lambda$. (This can be also seen from the proof below.) Thus, in view of Lemma 3.4, it will suffice to prove that for any $f \in \Pi_{n_{1}}^{d}$ with $\min _{\omega \in \Lambda} f(\omega) \geq 0, \ell(f) \geq 0$. To see this, we note that if $f(\omega) \geq 0$, then for any $x \in B_{\rho}\left(\omega, \frac{\delta}{n}\right)$,

$$
\begin{aligned}
2 f(x)-f(\omega) & \geq \max _{z \in B_{\rho}\left(\omega, \frac{\delta}{n}\right)}|f(z)|-\max _{z \in B_{\rho}\left(\omega, \frac{\delta}{n}\right)}(|f(z)|-f(\omega)-2 f(x)+2 f(\omega)) \\
& \geq \max _{z \in B_{\rho}\left(\omega, \frac{\delta}{n}\right)}|f(z)|-3 \max _{y \in B_{\rho}\left(\omega, \frac{\delta}{n}\right)}|f(y)-f(\omega)| .
\end{aligned}
$$

Thus, for $f \in \Pi_{n_{1}}^{d}$ with $\min _{\omega \in \Lambda} f(\omega) \geq 0$, we have

$$
\begin{aligned}
& \ell(f)=\sum_{\omega \in \Lambda} \int_{B_{\rho}\left(\omega, \frac{\delta}{n}\right)}(2 f(x)-f(\omega)) \frac{d \sigma(x)}{B(x)} \\
& \geq \sum_{\omega \in \Lambda}\left[\max _{z \in B_{\rho}\left(\omega, \frac{\delta}{n}\right)}|f(z)|-3 \max _{y \in B_{\rho}\left(\omega, \frac{\delta}{n}\right)}|f(y)-f(\omega)|\right] \int_{B_{\rho}\left(\omega, \frac{\delta}{n}\right)} \frac{d \sigma(x)}{B(x)} \\
& \geq \int_{B(e, \alpha)}|f(x)| d \sigma(x)-3 \sum_{\omega \in \Lambda}\left(\max _{y \in B_{\rho}\left(\omega, \frac{\delta}{n}\right)}|f(y)-f(\omega)|\right)\left|B_{\rho}\left(\omega, \frac{\delta}{n}\right)\right|,
\end{aligned}
$$

which, by (1.7) with $n$ replaced by $n_{1}$, is greater or equal

$$
\left(1-3 C_{1} \frac{n_{1} \delta}{n}\right) \int_{B(e, \alpha)}|f(x)| d \sigma(x) \geq \frac{1}{4} \int_{B(e, \alpha)}|f(x)| d \sigma(x) \geq 0 .
$$

This proves the claim (3.12).

Now setting

$$
\lambda_{\omega}=\frac{1}{2} \mu_{\omega}+\frac{1}{2} \int_{B_{\rho}(\omega, \delta / n)} \frac{d \sigma(x)}{B(x)}, \quad \omega \in \Lambda,
$$

and taking into account (3.11) and (3.12), we conclude that (1.1) with $\lambda_{\omega}$ satisfying the condition $\lambda_{\omega} \geq C^{-1}\left|B_{\rho}\left(\omega, \frac{\delta}{n}\right)\right|$ holds for all $f \in \Pi_{n_{1}}$. Thus, it remains to show the inequality

$$
\lambda_{\omega} \leq C\left|B_{\rho}\left(\omega, \frac{\delta}{n}\right)\right|, \quad \omega \in \Lambda,
$$

where $C>0$ depends only on $d$. To this end, we set $n_{2}=\left[n_{1} /(2 d+2)\right]$ and

$$
A_{n_{1}}(\cos t)=\gamma_{n_{2}}\left(\frac{\sin \left(n_{2}+\frac{1}{2}\right) t}{\sin \frac{t}{2}}\right)^{2 d+2}, \quad t \in[-\pi, \pi],
$$

where $\gamma_{n_{2}}$ is a positive constant chosen so that $A_{n_{1}}(1)=1$. Then it is easy to verify that

$$
\left|A_{n_{1}}(\cos t)\right| \leq C\left(1+n_{1}|t|\right)^{-2 d-2}, \quad t \in[-\pi, \pi] .
$$


Now for a fixed $\omega=\left(\omega_{1}, \cdots, \omega_{d}, \omega_{d+1}\right) \equiv\left(\omega^{\prime}, \omega_{d+1}\right) \in \Lambda$, we define

$$
\begin{aligned}
f_{n_{1}}(y)= & A_{n_{1}}\left(\frac{y^{\prime} \cdot \omega^{\prime}}{\sin ^{2} \alpha}+\frac{\sqrt{y_{d+1}^{2}-\cos ^{2} \alpha} \sqrt{\omega_{d+1}^{2}-\cos ^{2} \alpha}}{\sin ^{2} \alpha}\right) \\
& +A_{n_{1}}\left(\frac{y^{\prime} \cdot \omega^{\prime}}{\sin ^{2} \alpha}-\frac{\sqrt{y_{d+1}^{2}-\cos ^{2} \alpha} \sqrt{\omega_{d+1}^{2}-\cos ^{2} \alpha}}{\sin ^{2} \alpha}\right),
\end{aligned}
$$

where $y=\left(y_{1}, \cdots, y_{d}, y_{d+1}\right) \equiv\left(y^{\prime}, y_{d+1}\right) \in B(e, \alpha)$. Since $A_{n_{1}}$ is an algebraic polynomial of degree at most $n_{1}$ on $[-1,1]$, it follows that $f_{n_{1}} \in \Pi_{n_{1}}^{d}$. Note, on the other hand,

$$
\begin{aligned}
\arccos [ & {\left[\frac{y^{\prime} \cdot \omega^{\prime}}{\sin ^{2} \alpha} \pm \frac{\sqrt{y_{d+1}^{2}-\cos ^{2} \alpha} \sqrt{\omega_{d+1}^{2}-\cos ^{2} \alpha}}{\sin ^{2} \alpha}\right] } \\
& \sim \frac{1}{\sin \alpha} \sqrt{\left|y^{\prime}-\omega^{\prime}\right|^{2}+\left|\sqrt{y_{d+1}^{2}-\cos ^{2} \alpha} \mp \sqrt{\omega_{d+1}^{2}-\cos ^{2} \alpha}\right|^{2}} \\
& \geq \rho_{5}(y, \omega) \geq C \rho(y, \omega),
\end{aligned}
$$

where the last two inequalities follow by (2.21) and (2.22), respectively. Thus, by (3.14), we obtain

$$
0 \leq f_{n_{1}}(y) \leq C\left(1+n_{1} \rho(y, \omega)\right)^{-2 d-2}, \quad y \in B(e, \alpha) .
$$

Now applying the cubature formula (1.1) to $f_{n_{1}}$, we deduce

$$
\begin{aligned}
\lambda_{\omega} & \leq \lambda_{\omega} f_{n_{1}}(\omega) \leq \sum_{\xi \in \Lambda} \lambda_{\xi} f_{n_{1}}(\xi)=\int_{B(e, \alpha)} f_{n_{1}}(y) d \sigma(y) \\
& \leq C \sum_{j=0}^{\infty} \int_{\left\{y \in B(e, \alpha): \quad \frac{j}{n_{1}} \leq \rho(\omega, y) \leq \frac{j+1}{n_{1}}\right\}} f_{n_{1}}(y) d \sigma(y) \\
& \leq C\left|B_{\rho}\left(\omega, \frac{1}{n_{1}}\right)\right| \sum_{j=0}^{\infty}(j+1)^{-d-1} \leq C\left|B_{\rho}\left(\omega, \frac{\delta}{n}\right)\right|,
\end{aligned}
$$

which gives (3.13) and hence completes the proof of Corollary 1.2.

Proof of Corollary 1.3. Let $\mathcal{A}$ be a maximal $\left(\frac{1}{n}, \rho\right)$-separable subset of $B(e, \alpha)$. Then by Equation (1.7) and Lemma $2.2(\mathrm{v})$, it is easily seen that for $f \in \Pi_{n}^{d}$ and $1 \leq p<\infty$,

$$
\sum_{\xi \in \mathcal{A}}\left(\max _{x \in B_{\rho}\left(\xi, \frac{1}{n}\right)}|f(x)|^{p}\right)\left|B_{\rho}\left(\xi, \frac{1}{n}\right)\right| \leq C \int_{B(e, \alpha)}|f(x)|^{p} d \sigma(x) .
$$

Using this last fact, we obtain

$$
\begin{aligned}
& \sum_{\omega \in \Lambda}|f(\omega)|^{p}\left|B_{\rho}\left(\omega, \frac{1}{n}\right)\right| \leq \sum_{\xi \in \mathcal{A}} \sum_{\omega \in \Lambda \cap B_{\rho}\left(\xi, \frac{1}{n}\right)}|f(\omega)|^{p}\left|B_{\rho}\left(\omega, \frac{1}{n}\right)\right| \\
& \leq C \sum_{\xi \in \mathcal{A}}\left(\max _{x \in B_{\rho}\left(\xi, \frac{1}{n}\right)}|f(x)|^{p}\right) \#\left(\Lambda \cap B_{\rho}\left(\xi, \frac{1}{n}\right)\right)\left|B_{\rho}\left(\xi, \frac{1}{n}\right)\right| \\
& \leq C \tau \int_{B(e, \alpha)}|f(x)|^{p} d \sigma(x),
\end{aligned}
$$


where in the second inequality we have used the fact that $\left|B_{\rho}\left(\xi, \frac{1}{n}\right)\right| \sim\left|B_{\rho}\left(x, \frac{1}{n}\right)\right|$ whenever $x \in B_{\rho}\left(\xi, \frac{1}{n}\right)$, and in the last inequality we have used (3.15) and the definition of $\tau$. This completes the proof of Corollary 1.3.

Proof of Corollary 1.4. Corollary 1.4 is a simple consequence of Equation (1.7) and Lemma $2.2(\mathrm{v})$. We omit the detail.

Now it remains to show Lemma 3.5.

Proof of Lemma 3.5. Suppose that $f \in \Pi_{n}^{d}$ and $\Lambda$ is a $\left(\frac{\delta}{n}, \rho\right)$-separable subset of $B(e, \alpha)$. We set $\Lambda_{2}=\Lambda \cap B\left(e ; \frac{\alpha}{12}, \alpha\right)$. Since Lemma 3.5 is a direct consequence of Lemma 3.2 in the case when $d=1$, we shall assume $d \geq 2$ in the proof below. Also, without loss of generality we may assume that $e=(0,0, \cdots, 0,1) \in \mathbb{S}^{d}$.

Recall that $\rho_{4}$ is a metric on $B(e, \alpha)$ defined by (2.20). It follows by (2.23) and (2.26) with $\varepsilon=\frac{1}{24}$ that there exists an absolute constant $C_{3} \geq 1$ such that

$$
C_{3}^{-1} \rho(x, y) \leq \rho_{4}(x, y) \leq C_{3} \rho(x, y), \quad \text { for all } x, y \in B\left(e ; \frac{\alpha}{24}, \alpha\right),
$$

and

$$
B_{\rho_{4}}\left(x, C_{3}^{-1} r\right) \subset B_{\rho}(x, r) \subset B_{\rho_{4}}\left(x, C_{3} r\right) \text {, for all } x \in B\left(e ; \frac{\alpha}{24}, \alpha\right) \text { and } r>0 .
$$

Next, recall that $\rho_{1} \equiv \rho_{[-\alpha, \alpha]}$ is the metric on $[-\alpha, \alpha]$ defined by (2.1). Let $\left\{v_{i}\right\}_{i=0}^{L_{n}}$ be a sequence of numbers in $\left[\frac{\alpha}{12}, \alpha\right]$ satisfying the conditions $\min _{0 \leq i \neq j \leq L_{n}} \rho_{1}\left(v_{i}, v_{j}\right) \geq \frac{3^{-1} C_{3}^{-1} \delta}{n}$ and $\left[\frac{\alpha}{12}, \alpha\right] \subset \bigcup_{i=0}^{L_{n}} B_{\rho_{1}}\left(v_{i}, \frac{3^{-1} C_{3}^{-1} \delta}{n}\right)$. Let $\left\{\xi_{j}\right\}_{j=0}^{M_{n}}$ be a maximal $\left(\frac{3^{-1} C_{3}^{-1} \delta}{n}, d_{\mathbb{S}^{d-1}}\right)$-separable subset of $\mathbb{S}^{d-1}$, where $d_{\mathbb{S}^{d-1}}$ denotes the usual geodesic metric on $\mathbb{S}^{d-1}$. Set

$$
\omega_{i j}=\left(\xi_{j} \sin v_{i}, \cos v_{i}\right), \quad 0 \leq i \leq L_{n}, \quad 0 \leq j \leq M_{n} .
$$

Then it's easily seen that

$$
B\left(e ; \frac{\alpha}{12}, \alpha\right) \subset \bigcup_{i=0}^{L_{n}} \bigcup_{j=0}^{M_{n}} B_{\rho_{4}}\left(\omega_{i j}, \frac{3^{-1} C_{3}^{-1} \delta}{n}\right)
$$

On the other hand, by (3.16) it follows that $\Lambda_{2}$ is $\left(\frac{C_{3}^{-1} \delta}{n}, \rho_{4}\right)$-separable. This means that

$$
\#\left(\Lambda_{2} \bigcap B_{\rho_{4}}\left(\omega_{i j}, \frac{3^{-1} C_{3}^{-1} \delta}{n}\right)\right) \leq 1, \quad 0 \leq i \leq L_{n}, \quad 0 \leq j \leq M_{n} .
$$

Also, note that if $\omega \in \Lambda_{2} \cap B_{\rho_{4}}\left(\omega_{i j}, \frac{3^{-1} C_{3}^{-1} \delta}{n}\right)$, then by (3.17), (2.28) and (2.29), for any given $\beta \geq 1$,

$$
B_{\rho}\left(\omega, \frac{\beta \delta}{n}\right) \subset B_{\rho_{4}}\left(\omega_{i j}, \frac{C_{4} \delta}{n}\right), \text { and }\left|B_{\rho}\left(\omega, \frac{\delta}{n}\right)\right| \sim\left|B_{\rho_{4}}\left(\omega_{i j}, \frac{\delta}{n}\right)\right|,
$$

where $C_{4}=C_{3} \beta+3^{-1} C_{3}^{-1}$. 
Therefore, setting

$$
\mathcal{A}=\left\{(i, j) \in\left[0, L_{n}\right] \times\left[0, M_{n}\right]: \Lambda_{2} \bigcap B_{\rho_{4}}\left(\omega_{i j}, \frac{3^{-1} C_{3}^{-1} \delta}{n}\right) \neq \emptyset\right\}
$$

and taking into account (3.18) and (3.19), we conclude that for every $\omega \in \Lambda_{2}$, there exists a unique $(i, j) \in \mathcal{A}$ for which $\omega \in \Lambda_{2} \bigcap B_{\rho_{4}}\left(\omega_{i j}, \frac{3^{-1} C_{3}^{-1} \delta}{n}\right)$ and (3.20) holds. This implies

$$
\begin{aligned}
& \sum_{\omega \in \Lambda_{2}}\left(\max _{x, y \in B_{\rho}\left(\omega, \frac{\beta \delta}{n}\right)}|f(x)-f(y)|^{p}\right)\left|B_{\rho}\left(\omega, \frac{\delta}{n}\right)\right| \\
& \leq C \sum_{(i, j) \in \mathcal{A}}\left(\max _{x \in B_{\rho_{4}}\left(\omega_{i j}, \frac{C_{4} \delta}{n}\right)}\left|f(x)-f\left(\omega_{i j}\right)\right|^{p}\right)\left|B_{\rho_{4}}\left(\omega_{i j}, \frac{\delta}{n}\right)\right| \\
& \leq C \sum_{i=0}^{L_{n}} \sum_{j=0}^{M_{n}}\left(\max _{x \in B_{\rho_{4}}\left(\omega_{i j}, \frac{C_{4} \delta}{n}\right)}\left|f(x)-f\left(\omega_{i j}\right)\right|^{p}\right)\left|B_{\rho_{4}}\left(\omega_{i j}, \frac{\delta}{n}\right)\right| \equiv \Sigma .
\end{aligned}
$$

Thus, the proof of Lemma 3.5 is now reduced to the proof of the following inequality:

$$
\Sigma \leq(C \delta)^{p} \int_{B(e, \alpha)}|f(x)|^{p} d \sigma(x)
$$

where $\Sigma$ is defined by (3.21), and the constant $C$ depends only on $d, p$ and $\beta$.

For the rest of the proof, we shall write $\sum_{i, j}$ for $\sum_{i=0}^{L_{n}} \sum_{j=0}^{M_{n}}, \sum_{i}$ for $\sum_{i=0}^{L_{n}}$, and $\sum_{j}$ for $\sum_{j=0}^{M_{n}}$. Moreover, given $r>0$ and $\xi \in \mathbb{S}^{d-1}$, we denote by $B(\xi, r) \equiv B_{d_{\mathbb{S}^{d-1}}}(\xi, r)$ the spherical cap $\left\{\eta \in \mathbb{S}^{d-1}: \arccos \xi \cdot \eta \leq r\right\}$ in $\mathbb{S}^{d-1}$.

To show (3.22), we define $g(v, \eta)=f(\eta \sin v, \cos v)$, where $\eta \in \mathbb{S}^{d-1}$ and $v \in[-\alpha, \alpha]$, and we let $F$ be a polynomial on $\mathbb{R}^{d+1}$ of total degree at most $n$ whose restriction to $\mathbb{S}^{d}$ is $f$. Then, by the chain rule, we have, for $\eta=\left(\eta_{1}, \cdots, \eta_{d}\right) \in \mathbb{S}^{d-1}$,

$$
\frac{\partial g(v, \eta)}{\partial v}=\sum_{k=1}^{d} \frac{\partial F(\eta \sin v, \cos v)}{\partial x_{k}} \eta_{k} \cos v-\frac{\partial F(\eta \sin v, \cos v)}{\partial x_{d+1}} \sin v
$$

It follows that $\frac{\partial g(\cdot, \eta)}{\partial v} \in \Pi_{n}^{1}$ for each fixed $\eta \in \mathbb{S}^{d-1}$, and $\frac{\partial g(v, \cdot)}{\partial v} \in \Pi_{n}^{d-1}$ for each fixed $v \in[-\alpha, \alpha]$.

Now for each $(i, j) \in\left[0, L_{n}\right] \times\left[0, M_{n}\right]$, we assume

$$
\max _{x \in B_{\rho_{4}}\left(\omega_{i j}, \frac{C_{4} \delta}{n}\right)}\left|f(x)-f\left(\omega_{i j}\right)\right|=\left|f\left(x_{i j}^{*}\right)-f\left(\omega_{i j}\right)\right|,
$$


where $x_{i j}^{*}=\left(\xi_{i j}^{*} \sin \theta_{i j}^{*}, \cos \theta_{i j}^{*}\right) \in B_{\rho_{4}}\left(\omega_{i j}, \frac{C_{4} \delta}{n}\right)$, that is, $\xi_{i j}^{*} \in B\left(\xi_{j}, \frac{C_{4} \delta}{n}\right), \theta_{i j}^{*} \in B_{\rho_{1}}\left(v_{i}, \frac{C_{4} \delta}{n}\right)$. Then we have

$$
\begin{aligned}
& \left|f\left(x_{i j}^{*}\right)-f\left(\omega_{i j}\right)\right|^{p}=\left|g\left(\theta_{i j}^{*}, \xi_{i j}^{*}\right)-g\left(v_{i}, \xi_{j}\right)\right|^{p} \\
& \leq 2^{p}\left|g\left(v_{i}, \xi_{j}\right)-g\left(v_{i}, \xi_{i j}^{*}\right)\right|^{p}+2^{p}\left|g\left(v_{i}, \xi_{i j}^{*}\right)-g\left(\theta_{i j}^{*}, \xi_{i j}^{*}\right)\right|^{p} \\
& \leq 2^{p} \max _{\eta \in B\left(\xi_{j}, \frac{C_{4} \delta}{n}\right)}\left|g\left(v_{i}, \eta\right)-g\left(v_{i}, \xi_{j}\right)\right|^{p}+2^{p}\left|B_{\rho_{1}}\left(v_{i}, \frac{C_{4} \delta}{n}\right)\right|^{p-1} \int_{B_{\rho_{1}}\left(v_{i}, \frac{C_{4} \delta}{n}\right)}\left|\frac{\partial g\left(v, \xi_{i j}^{*}\right)}{\partial v}\right|^{p} d v \\
& \leq 2^{p} \max _{\eta \in B\left(\xi_{j}, \frac{C_{4} \delta}{n}\right)}\left|g\left(v_{i}, \eta\right)-g\left(v_{i}, \xi_{j}\right)\right|^{p}+C\left|B_{\rho_{1}}\left(v_{i}, \frac{\delta}{n}\right)\right|^{p-1} \int_{B_{\rho_{1}}\left(v_{i}, \frac{C_{4} \delta}{n}\right)}\left|\frac{\partial g\left(v, \xi_{j}\right)}{\partial v}\right|^{p} d v \\
& \quad+C\left|B_{\rho_{1}}\left(v_{i}, \frac{\delta}{n}\right)\right|^{p-1} \int_{B_{\rho_{1}}\left(v_{i}, \frac{C_{4} \delta}{n}\right)}\left(\max _{\eta \in B\left(\xi_{j}, \frac{C_{4} \delta}{n}\right)}\left|\frac{\partial g\left(v, \xi_{j}\right)}{\partial v}-\frac{\partial g(v, \eta)}{\partial v}\right|^{p}\right) d v \\
& \equiv A_{i j}+B_{i j}+C_{i j},
\end{aligned}
$$

where in the second inequality we have used Hölder's inequality and the fact that $B_{\rho_{1}}\left(v_{i}, \frac{C_{4} \delta}{n}\right)$ is a subinterval of $[-\alpha, \alpha]$ containing both $v_{i}$ and $\theta_{i j}^{*}$, and in the third inequality we have used (2.5) . Since, for any $\delta^{\prime} \in(0, \delta), \Lambda_{2}$ is, again, $\left(\rho, \frac{\delta^{\prime}}{n}\right)$-separable, without loss of generality, we may assume $\delta \in\left(0, \frac{1}{24 C_{4}}\right)$. Thus, by Lemma 2.1 (ii), we deduce

$$
B_{\rho_{1}}\left(v, \frac{C_{4} \delta}{n}\right) \subset\left[v-\frac{C_{4} \delta \alpha}{n}, \alpha\right] \subset\left[\frac{\alpha}{24}, \alpha\right], \quad \text { for any } v \in\left[\frac{\alpha}{12}, \alpha\right] .
$$

Hence, for each $(i, j)$, we have

$$
\left|B_{\rho_{4}}\left(\omega_{i j}, \frac{\delta}{n}\right)\right|=C_{d}\left|B\left(\xi_{j}, \frac{\delta}{n}\right)\right| \int_{B_{\rho_{1}}\left(v_{i}, \frac{\delta}{n}\right)} \sin ^{d-1} \theta d \theta \sim\left(\frac{\delta \alpha}{n}\right)^{d-1}\left|B_{\rho_{1}}\left(v_{i}, \frac{\delta}{n}\right)\right| .
$$

It follows by (3.21) that

$$
\begin{aligned}
\Sigma \leq & C\left(\frac{\delta \alpha}{n}\right)^{d-1} \sum_{i, j} A_{i j}\left|B_{\rho_{1}}\left(v_{i}, \frac{\delta}{n}\right)\right|+C\left(\frac{\delta \alpha}{n}\right)^{d-1} \sum_{i, j} B_{i j}\left|B_{\rho_{1}}\left(v_{i}, \frac{\delta}{n}\right)\right| \\
& +C\left(\frac{\delta \alpha}{n}\right)^{d-1} \sum_{i, j} C_{i j}\left|B_{\rho_{1}}\left(v_{i}, \frac{\delta}{n}\right)\right| \\
\equiv & \Sigma_{1}+\Sigma_{2}+\Sigma_{3} .
\end{aligned}
$$

For the first sum $\Sigma_{1}$, we have

$$
\begin{aligned}
\Sigma_{1} & \leq C \alpha^{d-1} \sum_{i}\left|B_{\rho_{1}}\left(v_{i}, \frac{\delta}{n}\right)\right|\left[\left(\frac{\delta}{n}\right)^{d-1} \sum_{j} \max _{\eta \in B\left(\xi_{j}, \frac{C_{4} \delta}{n}\right)}\left|g\left(v_{i}, \eta\right)-g\left(v_{i}, \xi_{j}\right)\right|^{p}\right] \\
& \leq(C \delta)^{p} \sum_{i} \alpha^{d-1}\left|B_{\rho_{1}}\left(v_{i}, \frac{\delta}{n}\right)\right| \int_{\mathbb{S}^{d-1}}\left|g\left(v_{i}, \eta\right)\right|^{p} d \sigma(\eta) \\
& \leq(C \delta)^{p} \int_{\mathbb{S}^{d-1}}\left[\sum_{i}\left|g\left(v_{i}, \eta\right)\right|^{p} \int_{B_{\rho_{1}}\left(v_{i}, \frac{\delta}{n}\right)}\left|\sin ^{d-1} v\right| d v\right] d \sigma(\eta) \\
& \leq(C \delta)^{p} \int_{\mathbb{S}^{d-1}} \int_{-\alpha}^{\alpha}|g(v, \eta)|^{p}\left|\sin ^{d-1} v\right| d v d \sigma(\eta)=(C \delta)^{p} \int_{B(e, \alpha)}|f(x)|^{p} d \sigma(x),
\end{aligned}
$$


where in the second inequality we have used Lemma $3.3(\underline{3.6})$ and the fact that $g\left(v_{i}, \cdot\right) \in \Pi_{n}^{d-1}$ for each fixed $i$, in the third inequality we have used (3.23), and the last inequality follows by Lemma 3.2 and Lemma 2.1 (iv).

For the second sum $\Sigma_{2}$, we have

$$
\begin{aligned}
\Sigma_{2} & \leq C \alpha^{d-1} \sum_{i}\left|B_{\rho_{1}}\left(v_{i}, \frac{\delta}{n}\right)\right|^{p} \int_{B_{\rho_{1}}\left(v_{i}, \frac{C_{4} \delta}{n}\right)}\left[\left(\frac{\delta}{n}\right)^{d-1} \sum_{j}\left|\frac{\partial g\left(v, \xi_{j}\right)}{\partial v}\right|^{p}\right] d v \\
& \leq C \sum_{i} \int_{B_{\rho_{1}}\left(v_{i}, \frac{C_{4} \delta}{n}\right)}\left|\sin ^{d-1} v\right|\left|B_{\rho_{1}}\left(v, \frac{\delta}{n}\right)\right|^{p}\left(\int_{\mathbb{S}^{d-1}}\left|\frac{\partial g(v, \xi)}{\partial v}\right|^{p} d \sigma(\xi)\right) d v \\
& \leq C\left(\frac{\delta}{n}\right)^{p} \int_{-\alpha}^{\alpha}\left|\sin ^{d-1} v\right|\left(\frac{\alpha}{n}+\sqrt{\alpha^{2}-v^{2}}\right)^{p}\left(\int_{\mathbb{S}^{d-1}}\left|\frac{\partial g(v, \xi)}{\partial v}\right|^{p} d \sigma(\xi)\right) d v \\
& =C\left(\frac{\delta}{n}\right)^{p} \int_{\mathbb{S}^{d-1}}\left(\int_{-\alpha}^{\alpha}\left|\sin ^{d-1} v\right|\left(\frac{\alpha}{n}+\sqrt{\alpha^{2}-v^{2}}\right)^{p}\left|\frac{\partial g(v, \xi)}{\partial v}\right|^{p} d v\right) d \sigma(\xi) \\
& \leq(C \delta)^{p} \int_{\mathbb{S}^{d-1}}\left[\int_{-\alpha}^{\alpha}|g(v, \xi)|^{p}\left|\sin ^{d-1} v\right| d v\right] d \sigma(\xi)=(C \delta)^{p} \int_{B(e, \alpha)}|f(x)|^{p} d \sigma(x),
\end{aligned}
$$

where in the second inequality, we have used (3.23), (2.6), (3.5) and the fact that $\frac{\partial g(v, \cdot)}{\partial v} \in \Pi_{n}^{d-1}$ for each fixed $v$, in the third inequality, we have used (2.7) and (2.5), and in the last inequality, we have used (3.1) and the fact that $g(\cdot, \xi) \in \Pi_{n}^{1}$ for each fixed $\xi \in \mathbb{S}^{d-1}$.

For the third sum $\Sigma_{3}$, we have

$$
\begin{aligned}
\Sigma_{3} \leq & C \alpha^{d-1} \sum_{i}\left|B_{\rho_{1}}\left(v_{i}, \frac{\delta}{n}\right)\right|^{p} \\
& \times \int_{B_{\rho_{1}}\left(v_{i}, \frac{C_{4} \delta}{n}\right)}\left[\left(\frac{\delta}{n}\right)^{d-1} \sum_{j}\left(\max _{\eta \in B\left(\xi_{j}, \frac{C_{4} \delta}{n}\right)}\left|\frac{\partial g\left(v, \xi_{j}\right)}{\partial v}-\frac{\partial g(v, \eta)}{\partial v}\right|^{p}\right)\right] d v \\
\leq & (C \delta)^{p}\left(\frac{\delta}{n}\right)^{p} \sum_{i} \int_{B_{\rho_{1}}\left(v_{i}, \frac{C_{4} \delta}{n}\right)}\left|\sin ^{d-1} v\right|\left(\frac{\alpha}{n}+\sqrt{\alpha^{2}-v^{2}}\right)^{p} \\
& \times\left(\int_{\mathbb{S}^{d-1}}\left|\frac{\partial g(v, \xi)}{\partial v}\right|^{p} d \sigma(\xi)\right) d v \\
\leq & C \delta^{p}\left(\frac{\delta}{n}\right)^{p} \int_{\mathbb{S}^{d-1}}\left(\int_{-\alpha}^{\alpha}\left|\frac{\partial g(v, \xi)}{\partial v}\right|^{p}\left|\sin ^{d-1} v\right|\left(\frac{\alpha}{n}+\sqrt{\alpha^{2}-v^{2}}\right)^{p} d v\right) d \sigma(\xi) \\
\leq & C \delta^{2 p} \int_{\mathbb{S}^{d-1}}\left(\int_{-\alpha}^{\alpha}|g(v, \xi)|^{p}\left|\sin ^{d-1} v\right| d v\right) d \sigma(\xi)=C \delta^{2 p} \int_{B(e, \alpha)}|f(x)|^{p} d \sigma(x),
\end{aligned}
$$

where in the second inequality we have used (3.23), (2.6), (2.5) (3.6) and the fact that $\frac{\partial g(v, \cdot)}{\partial v} \in$ $\Pi_{n}^{d-1}$ for a fixed $v$, in the third inequality we have used Lemma 2.1 (iv), and in the last inequality, we have used (3.1) and the fact that $g(\cdot, \xi) \in \Pi_{n}^{1}$ for each fixed $\xi \in \mathbb{S}^{d-1}$.

Now putting the above estimates together, and taking into account (3.24), we deduce the desired inequality (3.22), and hence complete the proof of Lemma 3.5. 


\section{Proofs of the Main Results For $\alpha \in\left[\frac{1}{2}, \pi\right)$}

Let $\varepsilon \in(0,1)$. In this section we shall prove Theorem 1.1 and Corollaries $1.2-1.4$ in the case when $\alpha \in\left[\frac{1}{2}, \pi-\varepsilon\right]$. It turns out that the main results in this case can be deduced from the already proven case $\alpha \in\left(0, \frac{1}{2}\right]$. Without loss of generality we may assume in this section that $d \geq 2$ and $e=(0, \cdots, 0,1) \in \mathbb{S}^{d}$. (The proof for the case $d=1$ is similar and in fact, much simpler.) For $x=(\eta \sin \theta, \cos \theta)$ with $\theta \in[0, \pi]$ and $\eta \in \mathbb{S}^{d-1}$, we define

$$
T x:=(\eta \sin (8 \theta), \cos (8 \theta)) \text {. }
$$

Then $T$ is a map from $B\left(e, \frac{\alpha}{8}\right)$ to $B(e, \alpha)$. Also, we set

$$
D(\cos \theta):=\frac{\sin ^{d-1}(8 \theta)}{\sin ^{d-1} \theta}, \quad \theta \in[0, \pi] .
$$

Then $D$ is an algebraic polynomial on $[-1,1]$ of degree $7(d-1)$.

We need two lemmas, the first of which can be stated as follows.

Lemma 4.1. Let $\alpha \in(0, \pi)$ and let $T$ be defined by $\sqrt{4.1})$. Then the following statements hold true:

(i) If $f \in \Pi_{n}^{d}$ then $f \circ T \in \Pi_{8 n}^{d}$.

(ii) If $f$ is an integrable function on $B(e, \alpha)$, and $D$ is the polynomial defined by (4.2), then we have

$$
\int_{B(e, \alpha)} f(x) d \sigma(x)=8 \int_{B\left(e, \frac{\alpha}{8}\right)} f(T x) D(x \cdot e) d \sigma(x) .
$$

Proof. We start with the proof of (i). Setting

$$
A(\cos \theta):=\cos 8 \theta \text { and } B(\cos \theta):=\frac{\sin (8 \theta)}{\sin \theta},
$$

we obtain that for $x=(\eta \sin \theta, \cos \theta) \equiv\left(x^{\prime}, x_{d+1}\right) \in \mathbb{S}^{d}$,

$$
f(T x)=f(\eta \sin (8 \theta), \cos (8 \theta))=f((\eta \sin \theta) B(\cos \theta), A(\cos \theta))=f\left(x^{\prime} B\left(x_{d+1}\right), A\left(x_{d+1}\right)\right) .
$$

Note, however, that $A$ is a polynomial on $[-1,1]$ of degree 8 , and $B$ is a polynomial on $[-1,1]$ of degree 7. Assertion (i) then follows.

Next, we show (ii). In fact, we have

$$
\begin{aligned}
\int_{B(e, \alpha)} f(x) d \sigma(x) & =C_{d} \int_{0}^{\alpha} \int_{\mathbb{S}^{d-1}} f(\eta \sin \theta, \cos \theta) d \sigma(\eta) \sin ^{d-1} \theta d \theta \\
& =8 C_{d} \int_{0}^{\alpha / 8} \int_{\mathbb{S}^{d-1}} f(\eta \sin (8 \theta), \cos (8 \theta)) d \sigma(\eta) \sin ^{d-1}(8 \theta) d \theta \\
& =8 C_{d} \int_{0}^{\alpha / 8} \int_{\mathbb{S}^{d-1}}(f \circ T)(\eta \sin \theta, \cos \theta) d \sigma(\eta) D(\cos \theta) \sin ^{d-1} \theta d \theta \\
& =8 \int_{B(e, \alpha / 8)} f(T x) D(x \cdot e) d \sigma(x),
\end{aligned}
$$

proving (4.3).

Let $T$ be the map from $B(e, \alpha / 8)$ to $B(e, \alpha)$ defined by (4.1) and let $T^{-1}$ denote its inverse. Given a subset $E$ of $B(e, \alpha)$, we write

$$
T^{-1}(E)=\{x \in B(e, \alpha / 8): T x \in E\} .
$$


Also, we recall that $\rho_{B(e, \alpha)}$ denotes the metric on $B(e, \alpha)$ defined by (1.4). For simplicity, we shall write $\rho_{\alpha}=\rho_{B(e, \alpha)}$ and $\rho_{\alpha / 8}=\rho_{B(e, \alpha / 8)}$.

Now our second lemma can be stated as follows.

Lemma 4.2. Let $\varepsilon \in(0,1)$ and $\alpha \in(0, \pi-\varepsilon]$. Then there exists a positive constant $C_{5}$ depending only on $d$ and $\varepsilon$ when $\varepsilon$ is small such that the following statements hold true:

(i) For any $x, y \in B(e, \alpha / 8)$,

$$
C_{5}^{-1} \rho_{\alpha / 8}(x, y) \leq \rho_{\alpha}(T x, T y) \leq C_{5} \rho_{\alpha / 8}(x, y) .
$$

(ii) For any $x \in B(e, \alpha)$ and $r>0$,

$$
B_{\rho_{\alpha / 8}}\left(T^{-1} x, C_{5}^{-1} r\right) \subset T^{-1}\left(B_{\rho_{\alpha}}(x, r)\right) \subset B_{\rho_{\alpha / 8}}\left(T^{-1} x, C_{5} r\right) .
$$

(iii) For any measurable subset $E$ of $B(e, \alpha)$,

$$
C_{5}^{-1}\left|T^{-1}(E)\right| \leq|E| \leq C_{5}\left|T^{-1}(E)\right| .
$$

(iv) For any $x \in B(e, \alpha)$ and $r \in(0,1)$,

$$
C_{5}^{-1} \triangle_{r, B(e, \alpha)}(x) \leq\left|B_{\rho_{\alpha}}(x, r)\right| \leq C_{5} \triangle_{r, B(e, \alpha)}(x),
$$

where $\triangle_{r, B(e, \alpha)}(x)$ is defined by (1.6).

Proof. (i) Let $x=(\eta \sin \theta, \cos \theta)$ and $y=(\xi \sin t, \cos t)$ with $\theta, t \in[0, \alpha / 8]$ and $\xi, \eta \in \mathbb{S}^{d-1}$. Then, by (2.31), we have

$$
d(T x, T y) \sim|\theta-t|+|\xi-\eta| \sqrt{\sin (8 \theta) \sin (8 t)} \sim d(x, y) .
$$

Thus, it follows by (1.4) that

$$
\begin{aligned}
\rho_{\alpha}(T x, T y) & \sim \frac{d(T x, T y)}{\alpha}+\frac{|\sqrt{\alpha-8 \theta}-\sqrt{\alpha-8 t}|}{\sqrt{\alpha}} \\
& \sim \frac{d(x, y)}{\alpha}+\frac{\left|\sqrt{\frac{\alpha}{8}-\theta}-\sqrt{\frac{\alpha}{8}-t}\right|}{\sqrt{\alpha}} \sim \rho_{\alpha / 8}(x, y),
\end{aligned}
$$

which proves Assertion (i).

(ii) Assertion (ii) follows directly from Assertion (i).

(iii) Let $E$ be a measurable subset of $B(e, \alpha)$. Then using (4.3), we obtain

$$
|E|=8 \int_{T^{-1}(E)} D(x \cdot e) d \sigma(x) .
$$

Assertion (iii) then follows by noticing that $D(\cos \theta) \sim 1$ whenever $\theta \in\left[0, \frac{\pi-\varepsilon}{8}\right]$.

(iv) Assertion (iv) is a simple consequence of Assertions (ii) and (iii), Lemma 2.2 (iii) and the fact that $\triangle_{r, B\left(e, \frac{\alpha}{8}\right)}\left(T^{-1} x\right) \sim \triangle_{r, B(e, \alpha)}(x)$ for any $x \in B(e, \alpha)$ and $r \in(0,1)$.

Now we are in a position to prove Theorem 1.1 and Corollaries $1.2-1.4$ in the case when $\alpha \in\left[\frac{1}{2}, \pi-\varepsilon\right]$. 
Proof of Theorem 1.1. Suppose that $\Lambda$ is $\left(\rho_{\alpha}, \frac{\delta}{n}\right)$-separable in $B(e, \alpha)$. It then follows by Lemma 4.2 (i) that $T^{-1}(\Lambda)$ is $\left(\rho_{\alpha / 8}, \frac{\delta}{n C_{5}}\right)$-separable in $B(e, \alpha / 8)$. Thus, for any $\beta \geq 1$,

$$
\begin{aligned}
& \sum_{\omega \in \Lambda}\left(\max _{x, y \in B_{\rho_{\alpha}}\left(\omega, \frac{\delta}{n}\right)}|f(x)-f(y)|^{p}\right)\left|B_{\rho_{\alpha}}\left(\omega, \frac{\delta}{n}\right)\right| \\
& \quad \leq C_{5} \sum_{\omega \in \Lambda}\left(\max _{u, v \in T^{-1}\left(B_{\rho_{\alpha}}\left(\omega, \frac{\delta}{n}\right)\right)}|f(T u)-f(T v)|^{p}\right)\left|T^{-1}\left(B_{\rho_{\alpha}}\left(\omega, \frac{\delta}{n}\right)\right)\right| \\
& \quad \leq C_{5} \sum_{z \in T^{-1}(\Lambda)}\left(\max _{u, v \in B_{\rho_{\alpha / 8}}\left(z, \frac{C_{5} \delta}{n}\right)}|f(T u)-f(T v)|^{p}\right)\left|B_{\rho_{\alpha / 8}}\left(z, \frac{C_{5} \delta}{n}\right)\right| \\
& \quad \leq(C \delta)^{p} \int_{B(e, \alpha / 8)}|f(T x)|^{p} d \sigma(x) \leq(C \delta)^{p} \int_{B(e, \alpha)}|f(x)|^{p} d \sigma(x),
\end{aligned}
$$

where in the first inequality we have used Lemma 4.2 (iii), in the second inequality we have used Lemma 4.2 (ii), in the third inequality we have used the already proven case of Theorem 1.1 applied to $B(e, \alpha / 8)$ and the polynomial $f(T x) \in \Pi_{8 n}^{d}$, and in the last inequality we have used (4.3). This proves (1.7).

Proof of Corollary 1.2. Suppose $\Lambda$ is a maximal $\left(\rho_{\alpha}, \frac{\delta}{n}\right)$-separable subset of $B(e, \alpha)$. Then by Lemma 4.2 (i)-(ii), $T^{-1}(\Lambda)$ is $\left(\rho_{\alpha / 8}, \frac{C_{5}^{-1} \delta}{n}\right)$-separable in $B(e, \alpha / 8)$ and

$$
\bigcup_{\omega \in \Lambda} B_{\rho_{\alpha / 8}}\left(T^{-1} \omega, \frac{C_{5} \delta}{n}\right)=B(e, \alpha / 8) .
$$

Thus, slightly modifying the proof of Corollary 1.2 in the case $\alpha \in\left(0, \frac{1}{2}\right]$ given in Section 3 , we conclude that there exists a constant $\delta_{1} \in(0,1)$ depending only on $d$ such that if $\delta \in\left(0, C_{5}^{-1} \delta_{1}\right)$ then there exists a sequence of positive numbers $\mu_{\omega}, \quad \omega \in \Lambda$ such that

$$
\mu_{\omega} \sim \triangle_{\frac{\delta}{n}, B(e, \alpha / 8)}\left(T^{-1} \omega\right) \sim \triangle_{\frac{\delta}{n}, B(e, \alpha)}(\omega), \quad \omega \in \Lambda
$$

and such that for any $P \in \Pi_{8(n+d)}^{d}$,

$$
\int_{B(e, \alpha / 8)} P(y) d \sigma(y)=\sum_{\omega \in \Lambda} \mu_{\omega} P\left(T^{-1} \omega\right)
$$

It then follows by Lemma 4.1 that for any $f \in \Pi_{n}^{d}$,

$$
\int_{B(e, \alpha)} f(y) d \sigma(y)=8 \int_{B(e, \alpha / 8)} f(T y) D(y \cdot e) d \sigma(y)=\sum_{\omega \in \Lambda}\left(8 \mu_{\omega} D\left(e \cdot T^{-1} \omega\right)\right) f(\omega)
$$

Now setting

$$
\lambda_{\omega}=8 \mu_{\omega} D\left(e \cdot T^{-1} \omega\right), \quad \omega \in \Lambda
$$

and noticing that $D(x \cdot e) \sim 1$ for $x \in B(e, \alpha / 8)$, we deduce Corollary 1.2 with $\delta_{0}=C_{5}^{-1} \delta_{1}$. 
Proofs of Corollaries 1.3 and 1.4. First, note that given $\beta \geq 1$ and an arbitrary $\left(\rho_{\alpha}, \frac{\delta}{n}\right)$ separable subset $\mathcal{A}$ of $B(e, \alpha)$, we have, for any $x \in B(e, \alpha)$,

$$
\begin{aligned}
\sum_{\xi \in \mathcal{A}} \chi_{B_{\rho_{\alpha}}(\xi, \beta \delta / n)}(x) & =\sum_{\xi \in \mathcal{A}} \chi_{T^{-1}\left(B_{\rho_{\alpha}}(\xi, \beta \delta / n)\right)}\left(T^{-1} x\right) \\
& \leq \sum_{\eta \in T^{-1}(\Lambda)} \chi_{B_{\rho_{\alpha / 8}}{ }_{\left(\eta, C_{5} \beta \delta / n\right)}}\left(T^{-1} x\right) \leq C_{\beta},
\end{aligned}
$$

where the first inequality follows by Lemma 4.2 (ii), and the second inequality follows by Lemma 2.2 (v) and Lemma 4.2 (i). Now the rest of the proofs are almost identical to those for the case $\alpha \in\left(0, \frac{1}{2}\right]$. We omit the details.

\section{CONCluding REMARKS}

5.1. Weighted inequalities on spherical caps. Let $\alpha \in\left(0, \frac{1}{2}\right]$ and let $e$ be a fixed point on $\mathbb{S}^{d}$. A weight function $W$ on $B(e, \alpha)$ is called a doubling weight if there exists a constant $L>0$, called doubling constant, such that for every $x \in B(e, \alpha)$ and $r \in(0,1)$,

$$
\int_{B_{\rho}(x, 2 r)} W(y) d \sigma(y) \leq L \int_{B_{\rho}(x, r)} W(y) d \sigma(y),
$$

where $\rho \equiv \rho_{B(e, \alpha)}$ is defined by (1.4). Associated with a weight function $W$ on $B(e, \alpha)$, we define

$$
W_{n}(x):=\frac{1}{\left|B_{\rho}\left(x, \frac{1}{n}\right)\right|} \int_{B_{\rho}\left(x, \frac{1}{n}\right)} W(y) d \sigma(y), \quad n=1,2, \cdots, \quad x \in B(e, \alpha) .
$$

It follows by (2.29) that for a doubling weight $W$ on $B(e, \alpha)$,

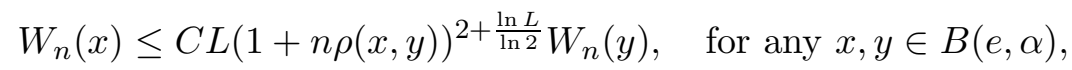

where $C$ is a constant depending only on $d$.

We have the following theorem:

Theorem 5.1. Let $e \in \mathbb{S}^{d}, 1 \leq p<\infty$ and $\alpha \in\left(0, \frac{1}{2}\right]$. Let $W$ be a doubling weight on $B(e, \alpha)$. Then for any $f \in \Pi_{n}^{d}$,

$$
\int_{B(e, \alpha)}|f(x)|^{p} W(x) d \sigma(x) \sim \int_{B(e, \alpha)}|f(x)|^{p} W_{n}(x) d \sigma(x),
$$

where the constant of equivalence depends only on $d, p$ and the doubling constant of $W$. Moreover, there exists a constant $\delta_{0}$ depending only on $d, p$, and the doubling constant of $W$ such that for any maximal $\left(\frac{\delta}{n}, \rho\right)$-separable subset $\Lambda$ of $B(e, \alpha)$ with $\delta \in\left(0, \delta_{0}\right)$, and any $f \in \Pi_{n}^{d}$, we have

$$
\begin{aligned}
\int_{B(e, \alpha)}|f(x)|^{p} W(x) d \sigma(x) & \sim \sum_{\omega \in \Lambda}\left(\max _{x \in B_{\rho}\left(\omega, \frac{\delta}{n}\right)}|f(x)|^{p}\right) \int_{B_{\rho}(\omega, \delta / n)} W(y) d \sigma(y) \\
& \sim \sum_{\omega \in \Lambda}\left(\min _{x \in B_{\rho}\left(\omega, \frac{\delta}{n}\right)}|f(x)|^{p}\right) \int_{B_{\rho}(\omega, \delta / n)} W(y) d \sigma(y),
\end{aligned}
$$

where the constants of equivalence depend only on $d, p$ and the doubling constant of $W$. 
Proof. For simplicity, associated with a function $f$ on $B(e, \alpha)$, we define

$$
\operatorname{osc}(f)(x, r)=\max _{y, z \in B_{\rho}(x, r)}|f(y)-f(z)|, \quad x \in B(e, \alpha), \quad r>0 .
$$

For the proof of Theorem 5.1, we claim that it is sufficient to prove that for any $\left(\frac{\delta}{n}, \rho\right)$ separable subset $\Lambda$ of $B(e, \alpha)$ and any $f \in \Pi_{n}^{d}$,

$$
\sum_{\omega \in \Lambda}|\operatorname{osc}(f)(\omega, \delta / n)|^{p} \int_{B_{\rho}\left(\omega, \frac{\delta}{n}\right)} W_{n}(y) d \sigma(y) \leq\left(C_{6} \delta\right)^{p} \int_{B(e, \alpha)}|f(x)|^{p} W_{n}(x) d \sigma(x),
$$

where $C_{6}$ depends only on $d, p$ and the doubling constant of $W$. In fact, once (5.5) is proved, then setting $\delta_{0}=\frac{1}{4 C_{6}}$, and taking into account Lemma $2.2(\mathrm{v})$, we conclude that for any maximal $\left(\frac{\delta_{0}}{n}, \rho\right)$-separable subset $\Lambda$ of $B(e, \alpha)$ and any $f \in \Pi_{n}^{d}$, we have

$$
\begin{aligned}
\int_{B(e, \alpha)}|f(x)|^{p} W_{n}(x) d \sigma(x) & \sim \sum_{\omega \in \Lambda}\left(\max _{x \in B_{\rho}\left(\omega, \frac{\delta_{0}}{n}\right)}|f(x)|^{p}\right) \int_{B_{\rho}\left(\omega, \delta_{0} / n\right)} W_{n}(y) d \sigma(y) \\
& \sim \sum_{\omega \in \Lambda}\left(\min _{x \in B_{\rho}\left(\omega, \frac{\delta_{0}}{n}\right)}|f(x)|^{p}\right) \int_{B_{\rho}\left(\omega, \delta_{0} / n\right)} W_{n}(y) d \sigma(y) .
\end{aligned}
$$

Equation (5.2) then follows by (5.6), (5.7), Lemma $2.2(\mathrm{v})$ and the doubling property of $W$. On the other hand, if $\Lambda$ is an arbitrary maximal $\left(\frac{\delta}{n}, \rho\right)$-separable subset of $B(e, \alpha)$ with $\delta \in\left(0, \delta_{0}\right)$, then setting $n_{1}=n \delta_{0} / \delta$, applying (5.2), (5.6) and (5.7) to $f \in \Pi_{n_{1}}^{d}$, and in view of Lemma 2.2 (v) and the doubling property of $W$, we deduce Equations (5.3) and (5.4).

Thus, it remains to prove (5.5). We sketch the proof as follows. First, we note that by (5.1) and the standard technique in [D1], there exists a sequence of positive polynomials $Q_{n} \in \Pi_{n}^{d}$ on $B(e, \alpha)$ such that $W_{n} \sim Q_{n}^{p}$ and

$$
\operatorname{osc}\left(Q_{n}\right)(x, \delta / n) \leq C \delta Q_{n}(x), \quad x \in B(e, \alpha) .
$$

It then follows that

$$
\begin{aligned}
W_{n}(\omega)\left(\operatorname{osc}(f)\left(\omega, \frac{\delta}{n}\right)\right)^{p} & \leq C\left(\operatorname{osc}\left(f Q_{n}\right)\left(\omega, \frac{\delta}{n}\right)\right)^{p}+C\left(\max _{y \in B_{\rho}\left(\omega, \frac{\delta}{n}\right)}|f(y)|^{p}\right)\left(\operatorname{osc}\left(Q_{n}\right)\left(\omega, \frac{\delta}{n}\right)\right)^{p} \\
& \leq C\left(\operatorname{osc}\left(f Q_{n}\right)\left(\omega, \frac{\delta}{n}\right)\right)^{p}+C \delta^{p} \max _{y \in B_{\rho}\left(\omega, \frac{\delta}{n}\right)}\left|f(y) Q_{n}(y)\right|^{p}
\end{aligned}
$$

which combined with Theorem 1.1 and Corollary 1.3 implies the desired inequality (5.5). This completes the proof.

Finally, we conjecture that (5.5) with $W_{n}$ replaced by $W$ remains true. Note that by Lemma 3.2 , this conjecture is true when $d=1$.

5.2. Analogous results on spherical collars. Let $e \in \mathbb{S}^{d}$ and $0<\alpha<\beta \leq \pi$. Recall that

$$
B(e ; \alpha, \beta)=\left\{x \in \mathbb{S}^{d}: \quad \alpha \leq d(x, e) \leq \beta\right\}
$$

denotes the spherical collar centered at $e$ of spherical height $\beta-\alpha$. We assume that $0<\alpha<$ $\beta<\pi-\varepsilon$ and $\alpha \sim \beta-\alpha$, where $\varepsilon \in(0,1)$ is a given absolute constant. We shall keep this assumption for the rest of this subsection. Without this assumption, some of the statements below may not be true. 
Associated with the spherical collar $B(e ; \alpha, \beta)$, we define

$$
\rho_{B(e ; \alpha, \beta)}(x, y):=\frac{1}{\alpha} \sqrt{|x-y|^{2}+\alpha\left|\sqrt{b_{x}}-\sqrt{b_{y}}\right|^{2}}, \quad x, y \in B(e ; \alpha, \beta),
$$

where $b_{x} \equiv b_{x, B(e ; \alpha, \beta)}$ denotes the shortest distance from $x \in B(e ; \alpha, \beta)$ to the boundary of $B(e ; \alpha, \beta)$, that is

$$
b_{x} \equiv b_{x, B(e ; \alpha, \beta)}:=\min \left\{d(x, y): \quad y \in \mathbb{S}^{d}, d(y, e)=\alpha \quad \text { or } \quad d(y, e)=\beta\right\} .
$$

It is easily seen that $\rho_{B(e ; \alpha, \beta)}$ is a metric on $B(e ; \alpha, \beta)$.

For $x=\xi \sin \theta+e \cos \theta$ and $y=\eta \sin t+e \cos t$ with $\xi, \eta \in \mathbb{S}_{e}^{d-1}$ and $\theta, t \in[\alpha, \beta]$, we define

$$
\rho_{6}(x, y):=\max \left\{|\xi-\eta|, \quad \rho_{[\alpha, \beta]}(\theta, t)\right\},
$$

where

$$
\rho_{[\alpha, \beta]}(\theta, t):=\frac{1}{\alpha} \sqrt{|\theta-t|^{2}+\alpha\left|\sqrt{b_{\theta,[\alpha, \beta]}}-\sqrt{b_{t,[\alpha, \beta]}}\right|^{2}}
$$

and $b_{u,[\alpha, \beta]}$ denotes the shortest distance from $u \in[\alpha, \beta]$ to the boundary of the interval $[\alpha, \beta]$, that is,

$$
b_{u,[\alpha, \beta]}:=\min \{|u-\alpha|, \quad|u-\beta|\} .
$$

It turns out that in the case $\alpha<\frac{\pi}{2}, \rho_{B(e ; \alpha, \beta)}$ and $\rho_{6}$ are equivalent on the whole spherical collar $B(e ; \alpha, \beta)$. (The proof of this fact is similar to that of Lemma 2.2 (i).)

Now our main results can be stated as follows:

Theorem 5.2. Let $\delta \in(0,1)$ and $1 \leq p<\infty$. Let $\rho \equiv \rho_{B(e ; \alpha, \beta)}$ be defined by (5.8) and let $\Lambda$ be $a\left(\frac{\delta}{n}, \rho\right)$-separable subset of $B(e ; \alpha, \beta)$. Then for all $f \in \Pi_{n}^{d}$, we have

$$
\sum_{\omega \in \Lambda}\left(\max _{x, y \in B_{\rho}\left(\omega, \frac{\delta}{n}\right)}|f(x)-f(y)|^{p}\right)\left|B_{\rho}(\omega, \delta / n)\right| \leq(C \delta)^{p} \int_{B(e ; \alpha, \beta)}|f(x)|^{p} d \sigma(x),
$$

where the constant $C$ depends only on $d$ and $p$.

Corollary 5.3. There exists a constant $\delta_{0} \in(0,1)$ depending only on $d$ such that for any $\delta \in\left(0, \delta_{0}\right)$ and any maximal $\left(\frac{\delta}{n}, \rho_{B(e ; \alpha, \beta)}\right)$-separable subset $\Lambda$ of $B(e ; \alpha, \beta)$ there exists a sequence of positive numbers $\lambda_{\omega}, \omega \in \Lambda$ for which the following cubature formula holds for all $f \in \Pi_{n}^{d}$ :

$$
\int_{B(e ; \alpha, \beta)} f(y) d \sigma(y)=\sum_{\omega \in \Lambda} \lambda_{\omega} f(\omega) .
$$

Results similar to Corollaries 1.3 and 1.4 can also be deduced from Theorem 5.2.

For the proofs of Theorems 5.2 and Corollary 5.3, the equivalence between the metrics $\rho_{B(e ; \alpha, \beta)}$ and $\rho_{6}$ plays an important role. Since the proofs run along the same lines as those of Theorem 1.1 and Corollary 1.2 given in Section 3, we omit the details.

\section{REFERENCES}

[BD] G. Brown and F. Dai, Approximation of smooth functions on compact two-point homogeneous spaces, $J$. of Funct. Anal. 220 (2005), 401-423.

[BDS] G. Brown, F. Dai and Y. Sh. Sun, Kolmogorov widths of classes of smooth functions on the sphere $\mathbb{S}^{d-1}$, J. Complexity 18 (2002), no. 4, 1001-1023.

[D1] F. Dai, Multivariate polynomial inequalities with respect to doubling weights and $A_{\infty}$ weights, J. Funct. Anal. 235 (2006), no. 1, 137-170. 
[D2] F. Dai, Characterizations of function spaces on the sphere using spherical frames, Trans. Amer. Math. Soc. 359 (2007), no. 2, 567-589.

[Er1] T. Erdélyi, Notes on inequalities with doubling weights, J. Approx. Theory 100 (1999), no. 1, 60-72.

[Er2] T. Erdélyi, Markov-Bernstein-type inequality for trigonometric polynomials with respect to doubling weights on $[-\omega, \omega]$, Constr. Approx. 19 (2003), no. 3, 329-338.

[GLN] L. Golinskii, D. S. Lubinsky, P. Nevai, Large sieve estimates on arcs of a circle, J. Number Theory 91 (2001), no. 2, 206-229.

[GS] T. Le Gia and I. H. Sloan, The uniform norm of hyperinterpolation on the unit sphere in an arbitrary number of dimensions, Constr. Approx. 17 (2001), no. 2, 249-265.

[KL1] C. K. Kobindarajah, D. S. Lubinsky, $L_{p}$ Markov-Bernstein inequalities on all arcs of the circle, J. Approx. Theory 116 (2002), no. 2, 343-368.

[KL2] C. K. Kobindarajah, D. S. Lubinsky, Marcinkiewicz-Zygmund type inequalities for all arcs of the circle, Advances in constructive approximation: Vanderbilt 2003, 255-264, Mod. Methods Math., Nashboro Press, Brentwood, TN, 2004.

[L] D. S. Lubinsky, $L_{p}$ Markov-Bernstein inequalities on arcs of the circle, J. Approx. Theory 108 (2001), no. $1,1-17$.

[MNW] H. N. Mhaskar, F. J. Narcowich and J. D. Ward, Spherical Marcinkiewicz-Zygmund inequalities and positive quadrature, Math. Comp. 70 (2001), 1113-1130 (Corrigendum: Math. Comp. 71 (2001) 453-454).

[M1] H. N. Mhaskar, Local quadrature formulas on the sphere, J. Complexity 20 (2004), no. 5, 753-772.

[M2] H. N. Mhaskar, On the representation of smooth functions on the sphere using finitely many bits, Appl. Comput. Harmon. Anal. 18 (2005), no. 3, 215-233.

[MT1] G. Mastroianni and V. Totik, Jackson type inequalities for doubling weights II, East J. Approx. 5 (1999), no. 1, 101-116.

[MT2] G. Mastroianni and V. Totik, Weighted polynomial inequalities with doubling and $A_{\infty}$ weights, Constr. Approx. 16 (2000), no. 1, 37-71.

[MT3] G. Mastroianni and V. Totik, Best approximation and moduli of smoothness for doubling weights, J. Approx. Theory 110 (2001), no. 2, 180-199.

[NPW1] F. J. Narcowich, P. Petrushev and J. D. Ward, Localized tight frames on spheres, SIAM J. Math. Anal. 38 (2006), no. 2, 574-594.

[NPW2] F. J. Narcowich, P. Petrushev and J. D. Ward, Decomposition of Besov and Triebel-Lizorkin spaces on the sphere, J. Funct. Anal. 238 (2006), no. 2, 530-564.

[Pe] P. Petrushev, Approximation by ridge functions and neural networks, SIAM J. Math. Anal. 30 (1999), no. 1, 155-189.

[Xu1] Yuan Xu, Orthogonal polynomials and cubature formulae on spheres and on balls, SIAM J. Math. Anal. 29(1998), no. 3, 779-793.

[Xu2] Yuan Xu, Orthogonal polynomials and cubature formulae on balls, simplices, and spheres, J. Comput. Appl. Math. 127 (2001), no. 1-2, 349-368.

Department of Mathematical and Statistical Sciences, University of Alberta, Edmonton, Alberta, T6G 2G1, Canada.

E-mail address: dfeng@math.ualberta.ca.

Department of Mathematics, Capital Normal University,, Beijing 100037, People's Republid OF CHINA. 